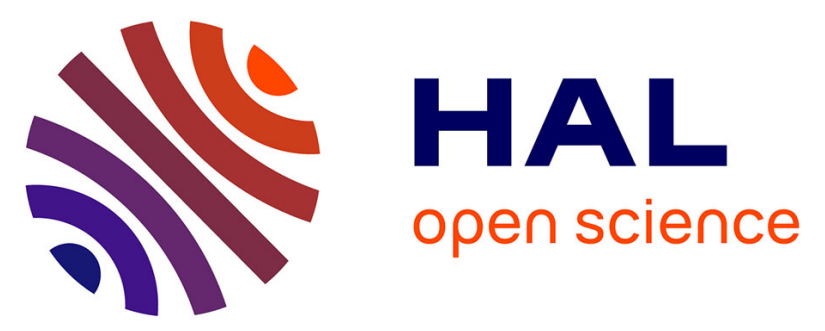

\title{
A novel hybrid surrogate model and its application on a mechanical system subjected to friction-induced vibration
}

\author{
Enora Denimal, L. Nechak, Jean-Jacques Sinou, Samuel Nacivet
}

\section{To cite this version:}

Enora Denimal, L. Nechak, Jean-Jacques Sinou, Samuel Nacivet. A novel hybrid surrogate model and its application on a mechanical system subjected to friction-induced vibration. Journal of Sound and Vibration, 2018, 434, pp.456-474. 10.1016/j.jsv.2017.08.005 . hal-02957474

HAL Id: hal-02957474

https://hal.inria.fr/hal-02957474

Submitted on 13 Jun 2021

HAL is a multi-disciplinary open access archive for the deposit and dissemination of scientific research documents, whether they are published or not. The documents may come from teaching and research institutions in France or abroad, or from public or private research centers.
L'archive ouverte pluridisciplinaire HAL, est destinée au dépôt et à la diffusion de documents scientifiques de niveau recherche, publiés ou non, émanant des établissements d'enseignement et de recherche français ou étrangers, des laboratoires publics ou privés. 
E. Denimal, L. Nechak, J-J. Sinou, S. Nacivet. A novel hybrid surrogate model and its application on a mechanical system subjected to friction-induced vibration. Journal of Sound and Vibration, 434 (2018), 456474. $10.1016 /$ j.jsv.2017.08.005

\title{
A novel hybrid surrogate model and its application on a mechanical system subjected to friction-induced vibration
}

\author{
Enora Denimal ${ }^{1,2, *}$, Lyes Nechak ${ }^{1}$, Jean-Jacques Sinou ${ }^{1,3}$, and Samuel Nacivet ${ }^{2}$ \\ ${ }^{1}$ Laboratoire de Tribologie et Dynamique des Systèmes, UMR CNRS 5513, École Centrale de Lyon, \\ 36 avenue Guy de Collongue 69134 Écully Cedex, France \\ ${ }^{2}$ PSA Peugeot Citroën, Centre technique de la Garenne Colombes, 18 rue des Fauvelles, \\ 92250 La Garenne Colombes, France \\ ${ }^{3}$ Institut Universitaire de France, 75005 Paris, France \\ *Corresponding authors: enora.denimal@inria.fr ; jean-jacques.sinou@ec-lyon.fr
}

\begin{abstract}
A new hybrid meta-modeling approach is proposed and developed for the propagating and quantifying of uncertainties in friction-induced instabilities. According to the available knowledge about design parameters, the associated uncertainty can be described by using different models. Hence, hybrid meta-models prove to be necessary to simultaneously treat different uncertainty models. So, this study presents a novel hybrid meta-model issued from the associating of the kriging formalism together with the generalized polynomial chaos for the prediction of friction-induced instabilities submitted to interval and probabilistic uncertainties. Its assessing through the considering of a friction system, reveals suitable accuracy about the estimating of the dispersion of the occurrences of instabilities. Moreover, it offers a promising alternative to the prohibitive Monte-Carlo/scanning based methods that are usually used for the same task.
\end{abstract}

Keywords Stability, Complex Eigenvalue Analysis, mode coupling, friction, meta-modeling, kriging, generalized polynomial chaos, uncertainty

\section{Introduction}

The uncertainty propagation, as well as the uncertainty quantification, in friction-induced instabilities are central steps helping for the setting up a robust design of mechanical systems. They are required in view of the non-negligible sensitivity of the stability property and, more generally, the dynamic behavior of selfexcited frictional system to the variations of design parameters. These facts have already been highlighted by several experimental and numerical studies. For example Butlin and Woodhouse $[1,2]$ demonstrated that friction-coupled systems are intrinsically sensitive to parameter variations and uncertainties. Oberst and Lai [3] also proposed an experimental approach to the statistical analysis of friction-induced instabilities in brake systems. Also, even if Culla and Massi [4] investigated contact instability under the influence of uncertainties via Monte Carlo simulation (MCS), it is now admitted that carrying out stability and non-linear self-excited friction-induced vibrations with uncertainties by using the conventional methods like scanning and/or Monte Carlo techniques is prohibitive and too costly in terms of computation time. Indeed, the use of such techniques requires high numbers of samples within the design space to ensure suitable accuracies and confidences of results.

Thus, one of the most important challenges today is to be able to considerably reduce the number of calculations to be carried out in order to predict friction-induced instabilities with uncertainties. In order to overcome this problem, strategies based on the searching for surrogate models, named 'meta-models', have been developed. A wide interest has been given to probabilistic meta-models and more particularly to the Generalized Polynomial Chaos (GPC) expansion to predict random friction-induced instabilities [5-7] when uncertain parameters are described by Probability Density Functions (PDF). When the probability distributions of uncertain parameters are not defined unambiguously (due to limited data of system parameters or the great number of experimental samples needed to perform statistics for example), it becomes more suitable to use 
non-probabilistic approaches including fuzzy methods [8] or interval analysis [9]. Another type of meta-model is based on kriging formalism [10, 11]. Such a meta-model approximates a parameter-dependent function by using a small number of parameter samples generated according to a predefined experimental design. Recently, kriging has been used successfully for the prediction of friction-induced instabilities [12-15].

Nowadays in the field of friction-induced instabilities, efforts have to be dedicated to the need for combine probabilistic uncertainty, due to the variability knowledge of some parameters, and intervals or fuzzy uncertainty, due to missing information or missing precision of other parameters. So, it becomes necessary to develop hybrid meta-models that are able to simultaneously propagate and quantify these uncertainties. In this context mixed uncertainty methods have also been proposed by several researchers [9, 16]. Otherwise, numerous software packages have been proposed to deal with meta-modeling for uncertainty propagation as well as for robust design optimization $[17,18]$.

In order to meet the challenge of combining aspects of interval analysis and probability theory in a way suitable for calculations of friction-induced instabilities, this paper proposes an hybrid meta-model based on the GPC expansion and the kriging formalism. Such an association has recently been proposed by Kersaudy et al. [19]. The main idea of their work consists in the replacing of the regressive part in the kriging meta-model by a GPC expansion. The approach presented here is quite different since it consists in the modeling of the random dimension with the GPC expansion and the dependence on the interval variables with the kriging. The determining of the hybrid meta-model is then turned into the problem of the evaluation of the coefficients of the GPC expansion by using kriging meta-models.

The paper is organized as follows. First, the kriging and GPC formalisms are presented in Section 2. Then the description of the mathematical formulation of the proposed hybrid meta-model is discussed. The mechanical model under study is briefly described in Section 3. Finally, efficiency and accuracy of the hybrid uncertainty propagation and quantification on the stability behaviour of the mechanical system under study is evaluated and commented.

\section{Meta-modelling methods}

This section is devoted to the description of the proposed hybrid meta-model issued from the association of the GPC expansion together with the kriging meta-model. The latter are first separately presented. The shared property of both meta-models is related to their non-intrusive calculation (i.e. there is no need to modify the system's equations). Only a small size set of learning data defined by a set of input/output samples generated according to a predefined plan of experiments, is required for the obtaining of the two meta-models and so the hybrid one.

\subsection{Mathematical formulation of kriging}

Let $y$ be a function in the vector of parameter $\mathbf{x} \in \mathbb{R}^{l}, l$ being the dimension of the design space. In the present study, $y$ represents the real part or the imaginary part of the complex eigenvalues of the mechanical system subjected to friction-induced vibrations (see Sections 3 and 4 for more details).

Based on kriging theory, the parameter-dependent function $y$ can be approximated by $Y$ defined from a regression model characterizing the average behavior of $\mathbf{y}$, one of which the dispersions are characterized by a spatial correlation function that measures how points in the design space are spatially close one to each other. This is formalized as follows:

$$
Y(\mathbf{x})=\mathbf{g}(\mathbf{x})^{\mathrm{T}} \boldsymbol{\beta}+\mathcal{Z}(\mathbf{x})
$$

where $\mathbf{g}(\mathbf{x})$ is the regression term composed of a $q$ basis polynomial functions $\mathbf{g}_{i}$ weighted by regression parameters $\boldsymbol{\beta}_{i}$. The second term $\mathcal{Z}($.$) is the realization of a zero-mean Gaussian process with a covariance matrix$ given by:

$$
\mathbb{E}[\mathcal{Z}(\mathbf{s}), \mathcal{Z}(\mathbf{x})]=\sigma^{2} \mathcal{R}(\boldsymbol{\theta}, \mathbf{s}, \mathbf{x})
$$

where $\sigma^{2}$ is the process variance and $\mathcal{R}(\boldsymbol{\theta}, \mathbf{s}, \mathbf{x}) \in[0,1]$ is the spacial correlation function with the scaling parameter $\boldsymbol{\theta} \in \mathbb{R}^{l}$ while $\mathbb{E}[$.] denotes the expectation operator. The correlation function is monotone and its construction is such that two identical points have a unitary correlation and two infinitely separated points have a zero correlation. Different choices of correlation functions are possible [17]. To construct a kriging model the regression parameters $\boldsymbol{\beta}$, the process variance $\sigma$ and the scaling parameters $\boldsymbol{\theta}$ have to be determined. This construction is strongly related to the experimental design set $\mathbf{S}=\left(\mathbf{s}^{(1)}, \ldots, \mathbf{s}^{(N)}\right)$ of $N$ sample points of the design space and the associated image $\mathbf{y}_{S}=\left(y\left(\mathbf{s}^{(1)}\right), \ldots, y\left(\mathbf{s}^{(N)}\right)\right)$. The parameter $\boldsymbol{\theta}$ is estimated from the solution of an optimization problem on the correlation matrix $\mathbf{R}$ defined as $R_{i j}=\mathcal{R}\left(\boldsymbol{\theta}, \mathbf{s}^{(i)}, \mathbf{s}^{(j)}\right)_{(i, j) \in[1, \ldots, N]^{2}}$ - Parameters $\boldsymbol{\beta}$ can then be written as the solution of a least square problem on the regression part of the 
problem. The process variance $\sigma^{2}$ is directly deduced from the previous results. The interested reader can find the details in [14].

An important step for the calculation of the kriging meta-model is then the generating of the learning data set. Different experimental designs may be considered. In this study, we have considered one of the most used experimental design, namely the Latin Hypercube Sampling (LHS) [14]. This plan is used together with the toolbox Matlab DACE developed for the the calculation of kriging meta-models [17].

\subsection{Mathematical formulation of Generalized Polynomial Chaos}

Let $Y(\boldsymbol{\xi})$ be a random-parameter function where $\boldsymbol{\xi}=\left(\xi_{1}, \ldots, \xi_{r}\right)$ is a vector of independent random variables $\xi_{i}$ with given $\mathrm{PDF} f_{i} . r$ corresponds to the stochastic dimension. According the the GPC theory [20-22], $Y$ can be approximate by a convergent, in the $\mathcal{L}^{2}$ sense, expansion:

$$
Y(\boldsymbol{\xi}) \approx \sum_{J=0}^{P-1} a_{J} \Psi_{J}(\boldsymbol{\xi})
$$

where $\left(\Psi_{J}\right)_{J \in \mathbb{N}}$ is the multivariate orthogonal polynomials and the weighting coefficients $\left(a_{J}\right)_{J \in \mathbb{N}}$ are the socalled stochastic modes of the random process. Elements of the multivariate polynomials are obtained by tensorization of an orthogonal polynomial sets $\left\{P_{j}^{i}\right\}_{j \in \mathbb{N}, i=1, \ldots, r}$ where $j$ is the order of $P_{j}^{i}$. Thus,

$$
\Psi_{J}(\boldsymbol{\xi})=\prod_{i=1}^{r} P_{J_{i}}^{i}\left(\xi_{i}\right)
$$

where $J=\sum_{i=1}^{r} J_{i}$ denotes the order of $\Psi_{J}$. Only polynomials with an order inferior or equal to the chaos order $m$ are kept. The number $P$ of polynomials is equal to $P=\left(\begin{array}{c}m+r \\ m\end{array}\right)$. The orthogonality is defined by the inner product:

$$
\left.<\Psi_{I}, \Psi_{J}>=\int \ldots \int \Psi_{I}(\boldsymbol{\xi}) \Psi_{J} \boldsymbol{\xi}\right) f(\boldsymbol{\xi}) \mathrm{d}(\boldsymbol{\xi})
$$

where $f(\boldsymbol{\xi})$ is the joint PDF. Polynomials $\left(P_{j}^{i}\right)$ are chosen according to the Askey-scheme [21, 22]. The latter defines an optimal correspondence between PDF and orthogonal polynomials families. For instance, Legendre and Jacobi polynomials prove to be optimal for uniform and beta distributions respectively. The calculation of coefficients $\left(a_{J}\right)$ can be made with intrusive and non-intrusive methods [23, 24]. As for the kriging meta-model, the non-intrusive scheme based on the regression method [25] is considered. The expansion coefficients $a_{J}$ are determined by minimizing in the least square sense the gap between the random function $Y(\boldsymbol{\xi})$ and its GPC approximation at $N$ points. To be solvable, the number of samples $N$ must be at least equal to the number $P$ of unknowns. In the case of a random experiment design or a LHS, taking $N=2 P$ leads to suitable results [26]. Another method consists in the constructing of an experimental design with roots of some polynomial with a suitable degree [25, 27-29]. In the one-dimension case $(r=1)$, this comes down to consider the roots $\left\{\alpha_{1}, \ldots, \alpha_{N}\right\}$ of a polynomial with an order $N \geq m+1$. In the multi-dimensional case, the experimental design is obtained by the tensor product of the roots defined in the one-dimensional case. However, its size given by $N=(m+1)^{r}$ may be very large when high chaos orders $m$ are required and/or in the case of problems with high stochastic dimensions $r$. Some methods have been developed to decrease the size of experiment designs, see for instance [29].

\subsection{Association of kriging and Generalized Polynomial Chaos Expansion}

Two different meta-models were previously presented. The GPC meta-model is suitable when parameters are characterized by probabilistic measures while the kriging meta-model can be used when only bounds of parameters are known. As mentioned in the introduction, there may be practical situations where the uncertainty of parameters is described by several models such as PDF, intervals, membership functions. In these cases, meta-models that are able to simultaneously deal with hybrid uncertainties are required. In this context, we propose an hybrid meta-model which associates the GPC and the kriging meta-models for the propagation and quantification of random and interval uncertainties. So, lets consider the two design spaces whose the dimensions are described by the interval parameters $\mathbf{x} \in \mathbb{R}^{l}$ and the random parameters $\boldsymbol{\xi}=\left(\xi_{1}, \ldots, \xi_{r}\right)$ defined previously. The parameter-dependent function is $Y(\mathbf{x}, \boldsymbol{\xi})$. The main objective is to define an hybrid meta-model which can suitably approximate the uncertain function $Y(\mathbf{x}, \boldsymbol{\xi})$. 
According to the GPC theory, $Y(\mathbf{x}, \boldsymbol{\xi})$ can be approximated by:

$$
Y(\mathbf{x}, \boldsymbol{\xi}) \simeq \sum_{J=0}^{P-1} a_{J}(\mathbf{x}) \Psi_{J}(\boldsymbol{\xi})
$$

where $\left(\Psi_{J}\right)_{J=0, \ldots, P-1}$ are the multivariate polynomials and $\left(a_{J}\right)_{J=1, \ldots, P-1}$ the associated stochastic modes that are in this case functions in the interval parameters $\mathbf{x}$.

In the same way and by using the kriging formalism, coefficients $\left(a_{J}\right)$ can be written as follows:

$$
a_{J}(\mathbf{x})=\mathbf{g}^{(J)}(\mathbf{x}) \boldsymbol{\beta}^{(J)}+\mathcal{Z}^{(J)}(\mathbf{x})
$$

where, as previously detailed in Section $2.1, \mathbf{g}^{(J)}$ is the $q$ basis polynomial functions $\mathbf{g}_{k}^{(J)}, \boldsymbol{\beta}^{(J)}$ the regression parameters and $\mathcal{Z}^{(J)}($.$) the realization of the zero-mean process associated to each parameter-dependent stochastic$ mode $\left(a_{J}\right)_{J=1, \ldots, P-1}$.

The meta-modeling of the uncertain function $Y(\mathbf{x}, \boldsymbol{\xi})$ is then turned into the problem of the estimation of the parameter-dependent stochastic modes $a_{J}(\mathbf{x})_{J=1, \ldots, P-1}$ of the the GPC expansion (6) by using the kriging meta-models. Ultimately, $Y(\mathbf{x}, \boldsymbol{\xi})$ can be approximate by the following double expansion:

$$
Y(\mathbf{x}, \boldsymbol{\xi}) \simeq \sum_{J=0}^{P-1}\left(\sum_{k=1}^{q} \boldsymbol{\beta}_{k}^{(J)} \mathbf{g}_{k}^{(J)}(\mathbf{x})+\mathcal{Z}^{(J)}(\mathbf{x})\right) \Psi_{J}(\boldsymbol{\xi})
$$

The main task lies in the estimation of the regression parameters $\boldsymbol{\beta}^{J}$, the variances $\sigma^{J}$ and the scaling parameters $\boldsymbol{\theta}^{(J)}$ for each coefficient $a_{J}(\mathbf{x})$. In this perspective, two learning data sets are required. The first one covering the random dimension is constructed by zeros of a polynomial with a suitable degree while the second, which is built from the LHS principle, covers the pavement defined by the interval parameters. Points $\mathbf{x}_{(i=1, \ldots, N)}^{(i)}$ within the second design experiment are used to determine a number of GPC expansions. Each of the latter is calculated by using a number of samples $\boldsymbol{\xi}_{(j=1, \ldots, Q)}^{(j)}$ within the first design experiment. Ultimately, a $(Q \times N)$ design experiment is required for the determining of the hybrid meta-model (8).

The main asset of this hybrid method presented here relies in the ability to deal with two kinds of uncertain parameters: either defined by a probability law, or by an interval.

\section{Mechanical model under study and stability analysis}

\subsection{Description of the phenomenological model}

The model under study is represented in Figure 1. This four-degrees-of-freedom is a phenomenological model that is an extension of the previous two-degree-of-freedom model proposed by Hulten [30]. Hulten initially introduced this simple model to study squeal vibration in drum brakes. In the present study, this four-degreesof-freedom allows to test the proposed hybrid meta-modeling technique on the problems of friction-induced vibrations for cases of different complexities. For the readers who are not specialist in the field of frictioninduced vibrations, we recall that the notion of friction-induced vibration corresponds to the phenomenon of sound and vibration generation by friction acting. The sound is generated due to the vibration of the self-excited mechanical system.

The proposed phenomenological model has already been discussed in [14] in order to investigate the case of multi-instabilities and to illustrate different stability behaviors characterized by mode coupling having coalescence patterns. Two specific numerical cases were investigated: the first one considers a simple mode coupling mechanism (i.e., coalescence of two modes, one being stable and the other unstable) while the second case deals with a more complex mode coupling mechanism characterized by successive appearances and disappearances of instabilities. These two different cases were considered in [14] to study the potential of kriging meta-models to be efficient predictors of friction-induced instabilities and so to be suitable alternative to the parameter-dependent Complex Eigenvalue Analysis (CEA).

The model consists of two masses $m_{1}$ and $m_{2}$ linearly coupled through a damper and an elastic spring whose damping and stiffness coefficients are given by $c_{a}$ and $k_{a}$ respectively. The two mass blocks are held against three moving bands as illustrated as in Figure 1. The belts run at a constant speed and the coefficient of friction $\mu$ at the surfaces of contact is constant. In the present study, the coefficient of friction $\mu$ is the same in all frictional interfaces for the sake of simplicity. Due to a preload applied on the system, it is assumed that masses and bands are always in contact. The classical Coulomb's law is used. Furthermore, it is assumed that the directions of tangential friction forces $F_{t}$ do not change since the relative velocities between the blocks 


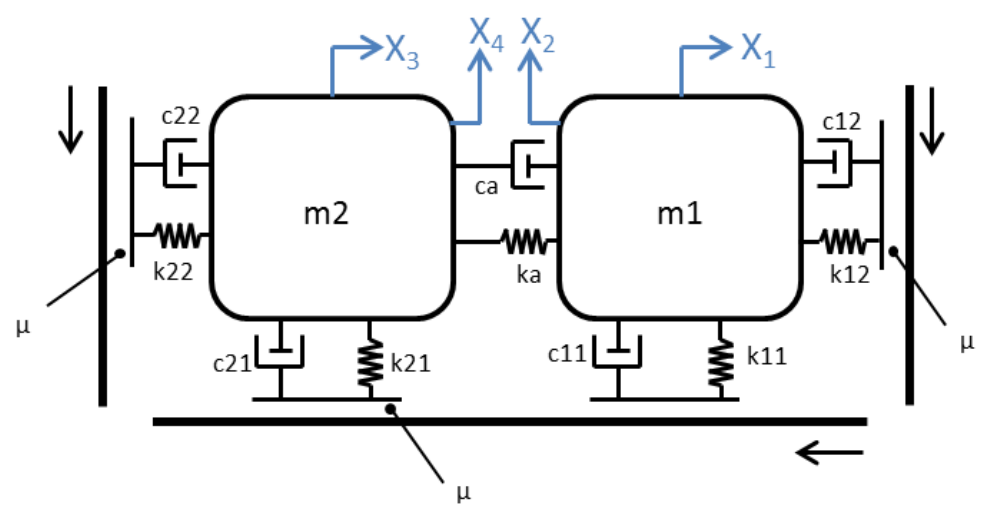

Figure 1: Mechanical model under study

and the belt are considered positive. So the tangential friction forces are proportional to the normal forces $F_{n}$, namely $F_{t}=\mu F_{n}$.

Lets denote $\mathbf{X}$ the displacement vector defined by $\mathbf{X}=\left(X_{1}, X_{2}, X_{3}, X_{4}\right)^{\mathrm{T}}$ and by a dot the temporal derivation. The equation of motion of the system can be expressed as follows:

$$
\mathbf{M} \ddot{\mathbf{X}}+\mathbf{C} \dot{\mathbf{X}}+\left(\mathbf{K}+\mathbf{K}_{\mu}\right) \mathbf{X}=\mathbf{0}
$$

where $\mathbf{M}, \mathbf{C}$ and $\mathbf{K}$ are the mass, damping and structural stiffness matrices of the mechanical system, respectively. The matrix $\mathbf{K}_{\mu}$ corresponds to the frictional contributions between the four plates and the three bands. It can be noted that the mass, damping and structural matrices are symmetric, while the stiffness matrix including the frictional contributions is non-symmetric. Expressions of the mass matrix $\mathbf{M}$, the damping matrix $\mathbf{C}$, the structural stiffness matrix $\mathbf{K}$ and the stiffness matrix $\mathbf{K}_{\mu}$ due to frictional forces are given by:

$$
\begin{gathered}
\mathbf{M}=\left[\begin{array}{cccc}
m_{1} & 0 & 0 & 0 \\
0 & m_{1} & 0 & 0 \\
0 & 0 & m_{2} & 0 \\
0 & 0 & 0 & m_{2}
\end{array}\right] \\
\mathbf{C}=\left[\begin{array}{cccc}
c_{12}+c_{a} & 0 & -c_{a} & 0 \\
0 & c_{11} & 0 & 0 \\
-c_{a} & 0 & c_{22}+c_{a} & 0 \\
0 & 0 & 0 & c_{21}
\end{array}\right] \\
\mathbf{K}=\left[\begin{array}{cccc}
k_{12}+k_{a} & 0 & -k_{a} & 0 \\
0 & k_{11} & 0 & 0 \\
-k_{a} & 0 & k_{22}+k_{a} & 0 \\
0 & 0 & 0 & k_{21}
\end{array}\right] \\
\mathbf{K}_{\mu}=\left[\begin{array}{cccc}
0 & \mu k_{11} & 0 & 0 \\
-\mu k_{12} & 0 & 0 & 0 \\
0 & 0 & 0 & \mu k_{21} \\
0 & 0 & -\mu k_{22} & 0
\end{array}\right]
\end{gathered}
$$

\subsection{Prediction of instabilities based on the Complex Eigenvalues Analysis}

The prediction of instabilities based on the CEA can be realized by achieving the eigenvalue analysis of the characteristic equation:

$$
\operatorname{det}\left(\lambda^{2} \mathbf{M}+\lambda \mathbf{C}+\mathbf{K}+\mathbf{K}_{\mu}\right)=0
$$

where $\lambda$ define the complex eigenvalues of the system. If all eigenvalues have negative real parts, the system is stable. If at least one eigenvalue has positive real part, the system is unstable. The imaginary part of the associated positive eigenvalue define the pulsation of the associated unstable mode. 


\begin{tabular}{|c|c|c|c|c|c|c|c|c|c|c|c|c|}
\hline Param. & $\begin{array}{l}\mathbf{m}_{1} \\
(\mathrm{~kg})\end{array}$ & $\begin{array}{l}\mathbf{m}_{2} \\
(\mathbf{k g})\end{array}$ & $\begin{array}{c}\mathbf{k}_{11} \\
\left(\mathbf{N} . \mathbf{m}^{-1}\right)\end{array}$ & $\begin{array}{c}\mathbf{k}_{12} \\
\left(\mathbf{N} \cdot \mathbf{m}^{-1}\right)\end{array}$ & $\begin{array}{c}\mathbf{k}_{21} \\
\left(\mathbf{N} . \mathbf{m}^{-1}\right)\end{array}$ & $\begin{array}{c}\mathbf{k}_{22} \\
\left(\mathbf{N} \cdot \mathbf{m}^{-1}\right)\end{array}$ & $\begin{array}{c}\mathbf{k}_{\mathbf{a}} \\
\left(\mathbf{N} \cdot \mathbf{m}^{-1}\right)\end{array}$ & $\begin{array}{c}\mathbf{c}_{11} \\
(\text { N.s.m } \\
\end{array}$ & $\begin{array}{c}\mathbf{c}_{12} \\
(\text { N.s.m } \\
\end{array}$ & $\begin{array}{c}\mathbf{c}_{21} \\
(\text { N.s.m } \\
\end{array}$ & $\begin{array}{c}\mathbf{c}_{22} \\
(\text { N.s.m } \\
-1\end{array}$ & $\begin{array}{c}c_{a} \\
(\text { N.s.m } \\
\end{array}$ \\
\hline Config. 1 & 1 & 1 & 3000 & 6000 & 1000 & 3000 & 100 & 1 & 1 & 1 & 1 & 1 \\
\hline Config. 2 & 1 & 1 & 3000 & 6000 & 1000 & 3000 & 1000 & 1 & 1 & 1 & 1 & 1 \\
\hline
\end{tabular}

Table 1: Set of parameters of the mechanical system for the two configurations under study

\subsection{Mode shape criterion to construct an efficient surrogate model}

The hybrid surrogate model presented in Section 2.3 is used in this paper to approximate the complex eigenvalues of the mechanical model under study (see Section 3.1). As previously discussed in [12, 14], the construction of a surrogate model for each separated mode needs to be able to follow the evolution of all separated eigenvalues versus the control parameters (i.e. the evolution of the real or imaginary parts versus the coefficient of friction for example). In the following, the Modal Assurance Criterion (MAC) that is one of the most efficient method to track accurately the evolution of a mode shape will be used to construct an hybrid surrogate model for the estimating of both the real and imaginary parts of eigenvalues and so for the predicting of hybrid parameterdependent mode coupling instabilities.

\section{Numerical Results}

In this section, the validity of the hybrid meta-model presented in Section 2.3 will be demonstrated. In this perspective, the proposed approach is used in order to model the eigenvalues of the mechanical system presented in Section 3. The final objective is to predict the stability behaviour of the system assuming several uncertain parameters. This section is constructed as follows: in a first part, the two reference configurations of the mechanical system are presented. Secondly the choice of the uncertain parameters and the different PDF that will be tested are also described. Then the hybrid method is applied by considering three uncertain parameters. Two of them are modelled with interval parameters (using the kriging formalism) and the third is modeled with a random parameter (using the GPC formalism). The efficiency of the proposed hybrid method will be demonstrated through various numerical simulations (for the two reference configurations of the mechanical system) and a comparison with the results obtained via the scanning/MCS. Finally, the effectiveness of the proposed methodology is investigated by considering an additional random parameter (i.e. four uncertain quantities will be considered for the numerical simulation).

\subsection{Reference configurations under study}

Two sets of parameters given in Table 1 are considered for the two reference configurations of the mechanical system under study. Results of the stability analysis with the evolution of real and imaginary parts versus the friction coefficient $\mu$ are presented in Figure 2. As previously explained in [14], the two studied configurations differ from each other with respect to the complexity of the phenomenon observed in the mode coupling modeling phenomena.

The first configuration can be considered as a classical baseline with "two simple mode coupling mechanisms". As illustrated in Figure 2(b) two independent coalescence patterns appear. For each coalescence one mode is unstable and the other one is stable. For the first coalescence, the instability is detected at $\mu=0.34$ and the pulsation of the associated unstable mode is 63.02 rad.s ${ }^{-1}$. And for the second coalescence, the instability appears at $\mu=0.58$ and the pulsation of the associated unstable mode is $41.66 \mathrm{rad} . \mathrm{s}^{-1}$.

The second configuration presents more complex coalescence patterns and more complex mode coupling mechanisms with crossing phenomenon between stable and unstable modes. The main origin of these behaviors is the high coupling between the two masses $m_{1}$ and $m_{2}$. Indeed, three different stages are observable as illustrated in Figures 2(c) and (d). A first instability appears for $\mu=0.20$, the pulsation of the associated

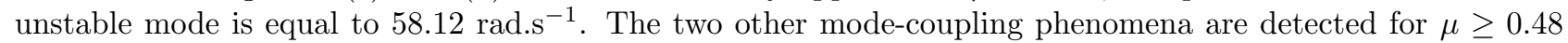
and $\mu \geq 0.81$. Frequencies of the associated unstable modes are $66.60 \mathrm{rad} . \mathrm{s}^{-1}$ and $44.73 \mathrm{rad} . \mathrm{s}^{-1} \mathrm{respectively.}$ Modes may be involved in several mode coupling coalescences. For instance, a crossing phenomenon is observed at $\mu=0.55$.

The main aim of investigating these two configurations in the following is to be able to demonstrate the efficiency of the proposed hybrid method for the prediction of classical and complex coalescence patterns. 


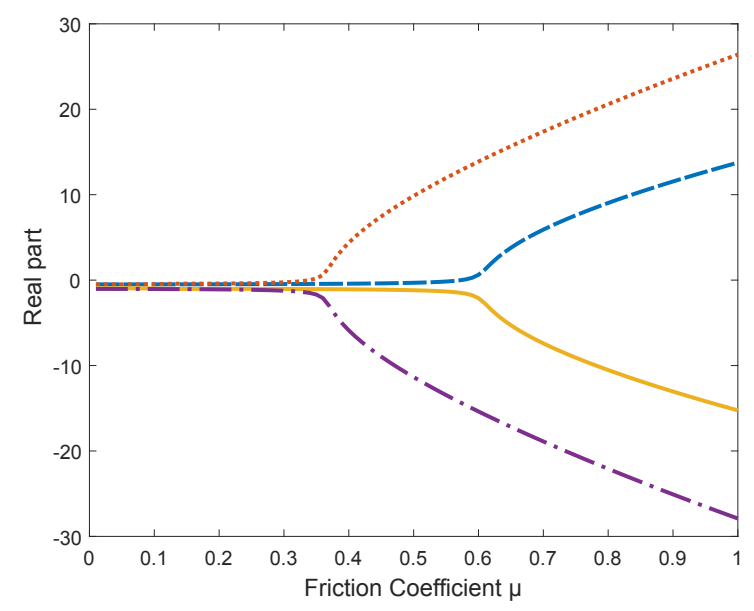

(a)

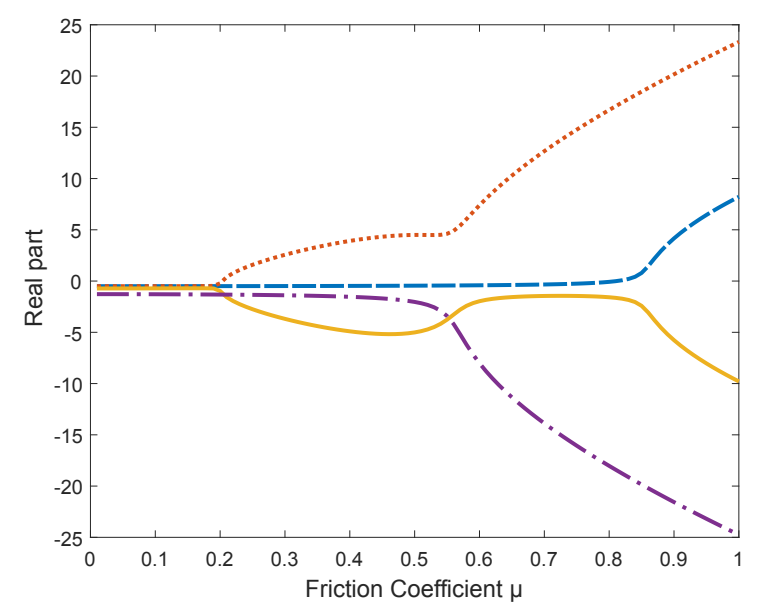

(c)

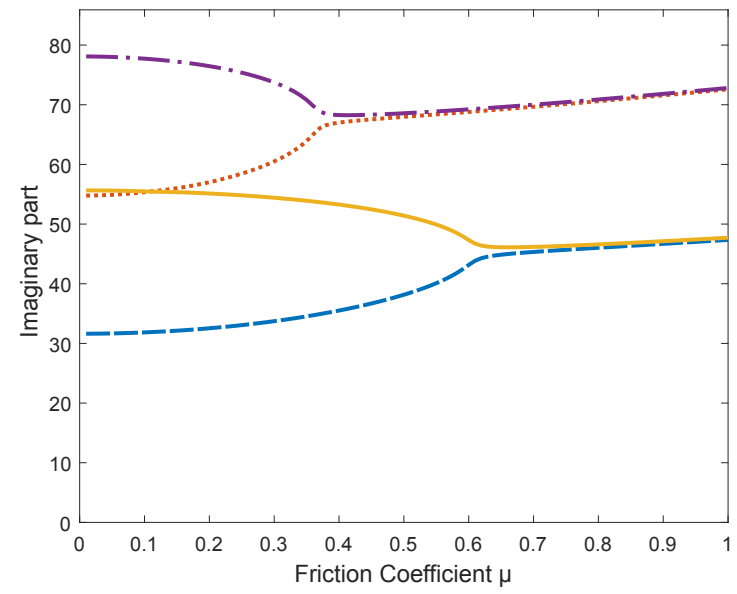

(b)

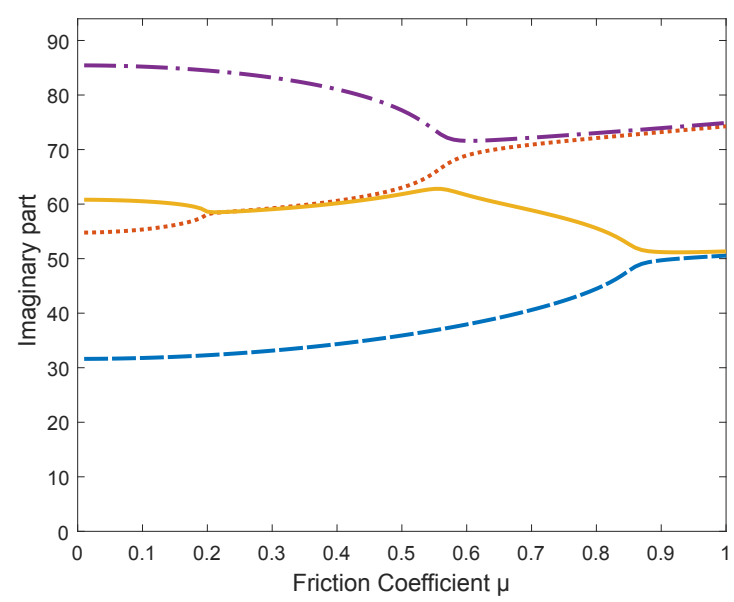

(d)

Figure 2: Evolution of real parts (a-c) and imaginary parts (b-d) of eigenvalues versus $\mu$ for configuration 1 (a-b) and configuration $2(\mathrm{c}-\mathrm{d})$

\subsection{Uncertain parameters}

The hybrid meta-model will be tested for different degrees of uncertainty on the two parameters: probabilistic description when the PDF is given and interval description when only bounds are known.

The types of uncertainty that will be considered in the following are defined by:

- The stiffness parameters $k_{11}$ and $k_{22}$ are considered within a given interval $\left[k_{11}^{\min } ; k_{11}^{\max }\right]$ and $\left[k_{22}^{\min } ; k_{22}^{\max }\right]$, respectively. We have:

$$
\begin{aligned}
& k_{11}=k_{11}^{\text {mean }}\left(1+0.1 \eta_{1}\right) \\
& k_{22}=k_{22}^{\text {mean }}\left(1+0.1 \eta_{2}\right)
\end{aligned}
$$

where $\eta_{1}$ and $\eta_{2}$ define two independent interval variables (with $\eta_{i} \in[-1 ; 1]$ for $i=1,2$ ).

- The friction coefficient $\mu$ and the stiffness $k_{a}$ are taken as random. They are defined as

$$
\begin{gathered}
\mu=\mu^{\text {mean }}\left(1+\delta_{\mu} \xi_{1}\right) \\
k_{a}=k_{a}^{\text {mean }}\left(1+\delta_{k_{a}} \xi_{2}\right)
\end{gathered}
$$

where $\xi_{1}$ and $\xi_{2}$ are random variables. $\delta_{\mu}$ and $\delta_{k_{a}}$ designates the variation coefficients of the friction value $\mu$ and the coupling stiffness, respectively. $\mu^{\text {mean }}$ and $k_{a}^{\text {mean }}$ are the mean values of coefficient of friction 


\begin{tabular}{cccc}
\hline Parameters & Min. value & Max. value & Types \\
\hline $\boldsymbol{\mu}$ & 0.1 & 0.9 & Random \\
$\mathbf{k}_{\mathbf{a}}$ & 100 & 1000 & Random \\
$\mathbf{k}_{\mathbf{1 1}}$ & 2700 & 3300 & Interval \\
$\mathbf{k}_{\mathbf{2 2}}$ & 2700 & 3300 & Interval \\
\hline
\end{tabular}

Table 2: Dispersion intervals of uncertain parameters

$\mu$ and the coupling stiffness, respectively. In the present study, two families of continuous probability distributions are considered: the uniform distribution and the beta distribution which are generally applied to model the behavior of random variables with bounded supports. Moreover the beta distribution can be all the way from approximately uniform to approximately normal. The beta distribution, defined on the unitary interval $[0 ; 1]$, is parametrized by two positive shape parameters; $\alpha$ and $\beta$ (denotes by $B(\alpha, \beta)$ ). The PDF of the beta distribution is a power function defined by:

$$
f(\xi ; \alpha, \beta)=\frac{\xi^{\alpha-1}(1-\xi)^{\beta-1}}{\int_{0}^{1} u^{\alpha-1}(1-u)^{\beta-1} \mathrm{~d} u} \text { for } \xi \in[0,1] ; \quad \alpha>0 \text { and } \beta>0
$$

The beta density function can take a wide variety of different shapes depending on the values of the two parameters $\alpha$ and $\beta$. Also, it may be noted that the beta distribution with $\alpha=\beta=1$ corresponds to the uniform $[0 ; 1]$ distribution. In the following three specific values of $\alpha$ and $\beta$ are chosen to investigate the influence of three different beta distributions:

$-\alpha=\beta=5$ or $B(5,5)$, symmetric with a skewness equal to 0 ,

$-\alpha=2$ and $\beta=8$ or $B(2,8)$, asymmetrical left-tailed curve/negative skew,

$-\alpha=8$ and $\beta=2$ or $B(8,2)$, asymmetrical right-tailed curve/positive skew.

The occurrences of the random parameter according to each distribution are displayed in Figure 3 . It may be noted that the shape of the beta distribution $B(5,5)$ looks as that of the normal distribution. The associated supports are different; unitary support for the beta distribution and infinite for the normal distribution. As indicated in Equations 17 and 18, the standard variables $\xi_{1}$ and $\xi_{2}$ are used to represent parameter uncertainties. A linear transformation maps the interval of each random variable $\left(\xi_{1}, \xi_{2}\right)$ to the interval of each parameter $\left(\mu, k_{a}\right)$, respectively (see Table 2 ).

\subsection{Case of three uncertain parameters: two interval and one random parameters}

In this section, three parameters are considered as uncertain: the two stiffnesses $k_{11}$ and $k_{22}$ and the friction coefficient $\mu$. As previously explained in Section 4.1, uncertainty of both stiffnesses $k_{11}$ and $k_{22}$ is modeled with intervals (see Equations 15 and 16, respectively) and the friction coefficient is supposed to be random by considering various beta distribution models (see Equation 17). All the other parameters are deterministic. The values of these deterministic parameters can be found in Table 1.

The hybrid meta-model is used to predict the stability behavior of the system in the case of the two configurations depicted previously. For each beta distribution model of the friction coefficient $\mu$ (while the other two uncertain parameters stiffnesses $k_{11}$ and $k_{22}$ are modelled with intervals), a meta-model is built to predict the system's eigenvalues (both real and imaginary parts) against the three parameters. Then, occurrences of the real and imaginary parts of unstable modes (i.e eigenmodes having eigenvalue with positive real part) are extracted and compared to the scanning/MCS which correspond to the reference results.

First of all the reference results are computed by performing a scanning/MCS with a regular grid of 2500 values for the parameters $\left(k_{11}-k_{22}\right)$ and by considering 10000 random values of the uncertain parameter $\mu$ generated according to its governing distributions. The occurrences of real and imaginary parts of unstable modes are based on these $2.510^{7} \mathrm{CEA}$ evaluations. These reference results are displayed in Figure 5 and 6 for configurations 1 and 2, respectively. For each configuration, the uniform $[0 ; 1]$ distribution and the three different beta distributions $(B(5,5), B(2,8)$ and $B(8,2))$ are tested. The repartition of real parts of unstable modes gives information about the instability growth rate. Histogram depicting the repartition of imaginary parts gives information about the unstable frequencies (i.e. that is the imaginary part of eigenvalues with positive real part). 

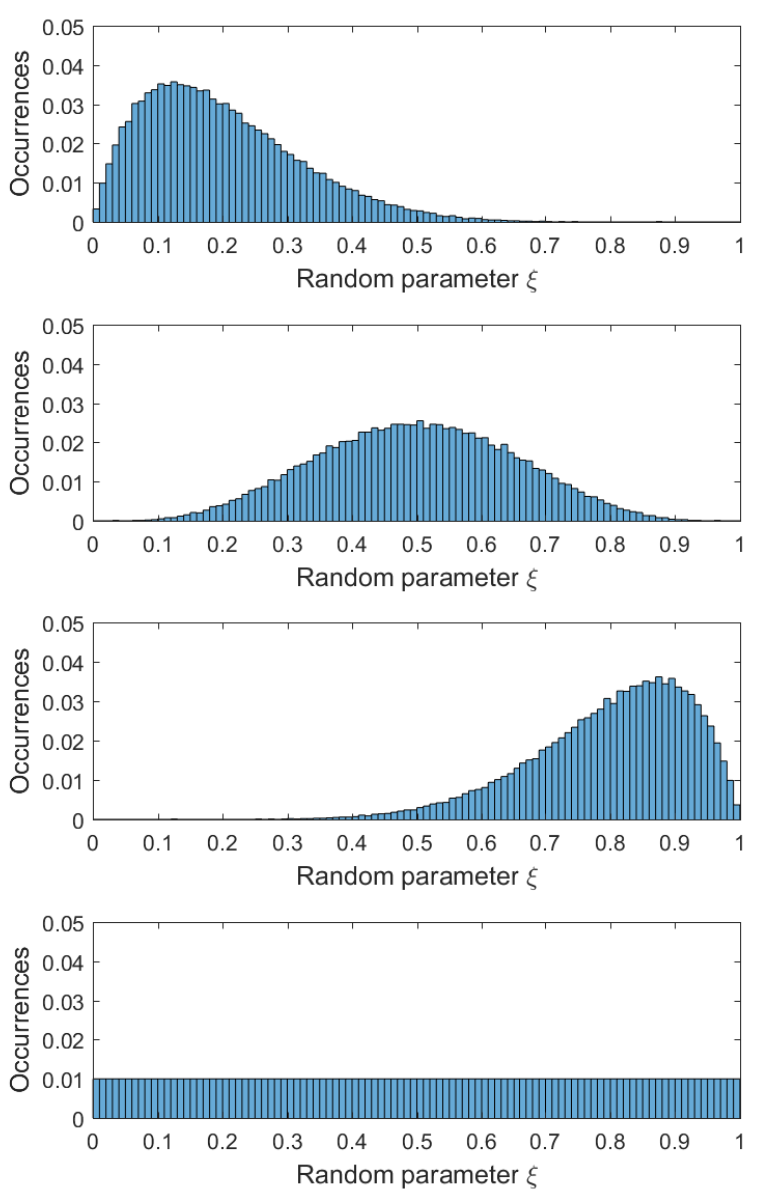

Figure 3: Probability density functions governing the random parameters - From top to bottom: $B(2,8)$ law, $B(5,5)$ law, $B(8,2)$ law and uniform law

Secondly, the proposed hybrid meta-model based on the GPC and the kriging meta-model is tested. The construction of this hybrid meta-model and its validation is performed in two steps. The first step concerns the calculation of the GPC coefficients via kriging meta-models and their validation. To built the GPC development, the Legendre and Jacobi polynomial basis are used in the case of the uniform and beta distributions, respectively. Based on a convergence study which is not presented here, the chaos order is fixed to 20 while the experimental design used for the calculation is composed of the roots of the $30^{\text {th }}$ polynomial of the used polynomial basis. Considering the beta distribution model of the friction coefficient, the random experimental design is composed of 30 sample points. Moreover, the GPC coefficients which are dependent on the variables $k_{11}$ and $k_{22}$ are approximated by kriging models with a cubic correlation function and a first order regression function. The $\left(k_{11}-k_{22}\right)$-experimental design used for the determining of the kriging meta-models consists in a LHS plan of 100 sample points. In conclusion the complete hybrid meta-model representing the eigenvalues depending on the interval parameters $\left(k_{11}-k_{22}\right)$ and the random parameter $\mu$ with the beta density functions, has required $30 * 100=3000$ CEA solutions. Figures 4 display the surface responses of the $1^{\text {st }}, 3^{\text {rd }}, 10^{\text {th }}, 15^{\text {th }}$ and $21^{\text {th }} \mathrm{GPC}$ coefficients for the real part of the first eigenvalue (i.e. the continuous one in the previous graphs) in the case of the first $B(5,5)$ distribution and for the first configuration. These results are plotted in black on Figures 4 . Sample points of the design space used to build the kriging surrogate models are represented in red. Results are compared with those of one baseline (plotted in blue) that are obtained by the construction of the GPC expansions for each $\left(k_{11}-k_{22}\right)$ value. The kriging models give faithful predictions of the GPC coefficients. Table 3 and Table 4 summarize the quadratic mean error committed on the first and second GPC coefficients for each real and imaginary part of eigenvalues for the first and the second distributions, respectively. The kriging based estimations of GPC coefficients are suitable since the maximal measured error is equal to $1.4110^{-2}$ for 

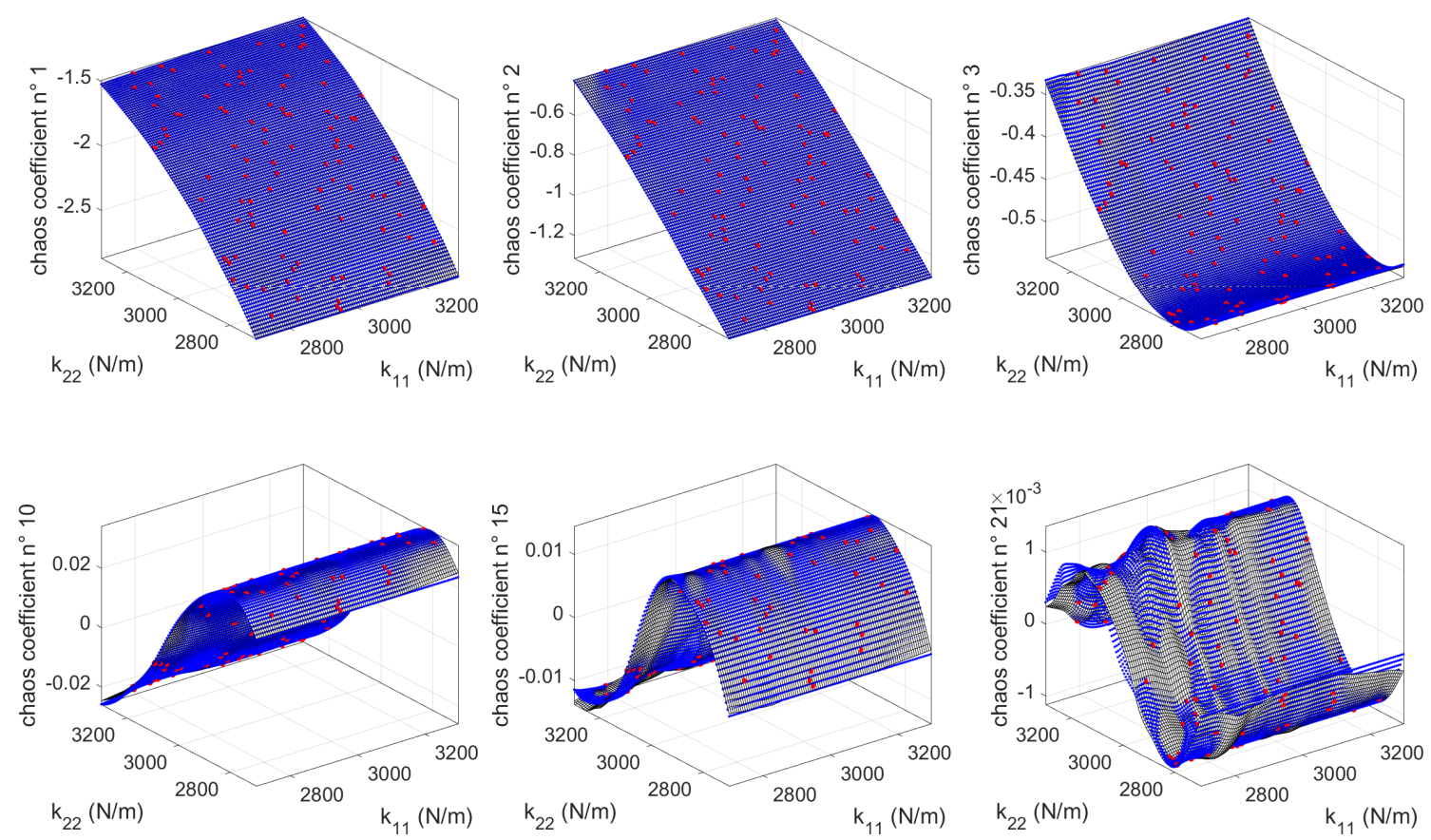

Figure 4: Comparison of the first, second, third, tenth, fifteenth and twenty-first chaos coefficients of the real part of an eigenvalue of the first configuration versus the stiffness coefficients $k_{11}$ and $k_{22}$

the real parts and $1.5210^{-2}$ for the imaginary parts. So, it may be concluded that the choice of modeling chaos coefficients via kriging surrogate models with the associated regression and correlation functions is suitable. The second step concerns the validation of the global hybrid meta-model based on the GPC and the kriging metamodel for the prediction of the occurrences of the real and imaginary parts of unstable modes. Histograms of real and imaginary parts of unstable mode occurrences are illustrated in Figures 5 and 6 by considering the uniform $[0 ; 1]$ distribution and the three different beta distributions $(B(5,5), B(2,8)$ and $B(8,2))$ for configurations 1 and 2 , respectively. It is very clear that the proposed hybrid meta-model reproduces very well the reference model in all cases. Moreover, Tables 5 and 6 depict the percentage of stable and unstable occurrences with specification of the number of unstable modes (for the first and second configurations, respectively). Here again, it is observed that the proposed hybrid meta-model gives a very suitable prediction of the propensity of instability.

Finally several additional comments can be done. All the numerical simulations show that the distribution law of the friction coefficient $\mu$ drastically modifies the percentage of stable and unstable occurrences with specification of the number of unstable modes (see Tables 5 and 6 ) as well as the instability growth rate (i.e. the positive real part) and the unstable frequencies (i.e. the imaginary part of eigenvalues with positive real part). For example, the simulation based on a $B(2,8)$ distribution returns a lower number of simultaneously unstable modes than the $B(5,5)$ or $B(8,2)$ distributions. The $B(2,8)$ distribution also increases the number of stable occurrences (see the percentage of stable and unstable occurrences in Tables 5 and 6 ). These results are of course expected since they illustrate the well-known role of the friction coefficient. Moreover, the propensity of instability notably varies when switching from a uniform distribution law to a beta distribution law (or by modifying the beta density function via the values of the two parameters $\alpha$ and $\beta$ ). Indeed unstable frequencies in the range $[40,50] \mathrm{Hz}$ that appear in the case of simulations using a uniform distribution law, the $B(5,5)$ or $B(8,2)$ distributions (a uniform distribution law or a $B(8,2)$ distribution, respectively) are not represented when considering a $B(2,8)$ distribution (the $B(5,5)$ or $B(2,8)$ distributions, respectively) for configuration 1 (configuration 2, respectively).

In most cases, it may be noted a significant change in the occurrence probability associated with each interval of unstable frequencies (i.e. around $[40,50] \mathrm{Hz}$ and $[60,75] \mathrm{Hz}$ for configuration 1; $[45,50] \mathrm{Hz},[55,65] \mathrm{Hz}$ and $[65,75] \mathrm{Hz}$ for configuration 2). For example, by considering a $B(2,8)$ distribution, there is only one unstable mode whose pulsation is between 60 and 70 rad.s ${ }^{-1}$. The pulsation is located around a peak at $68 \mathrm{rad} . \mathrm{s}^{-1}$ with a 

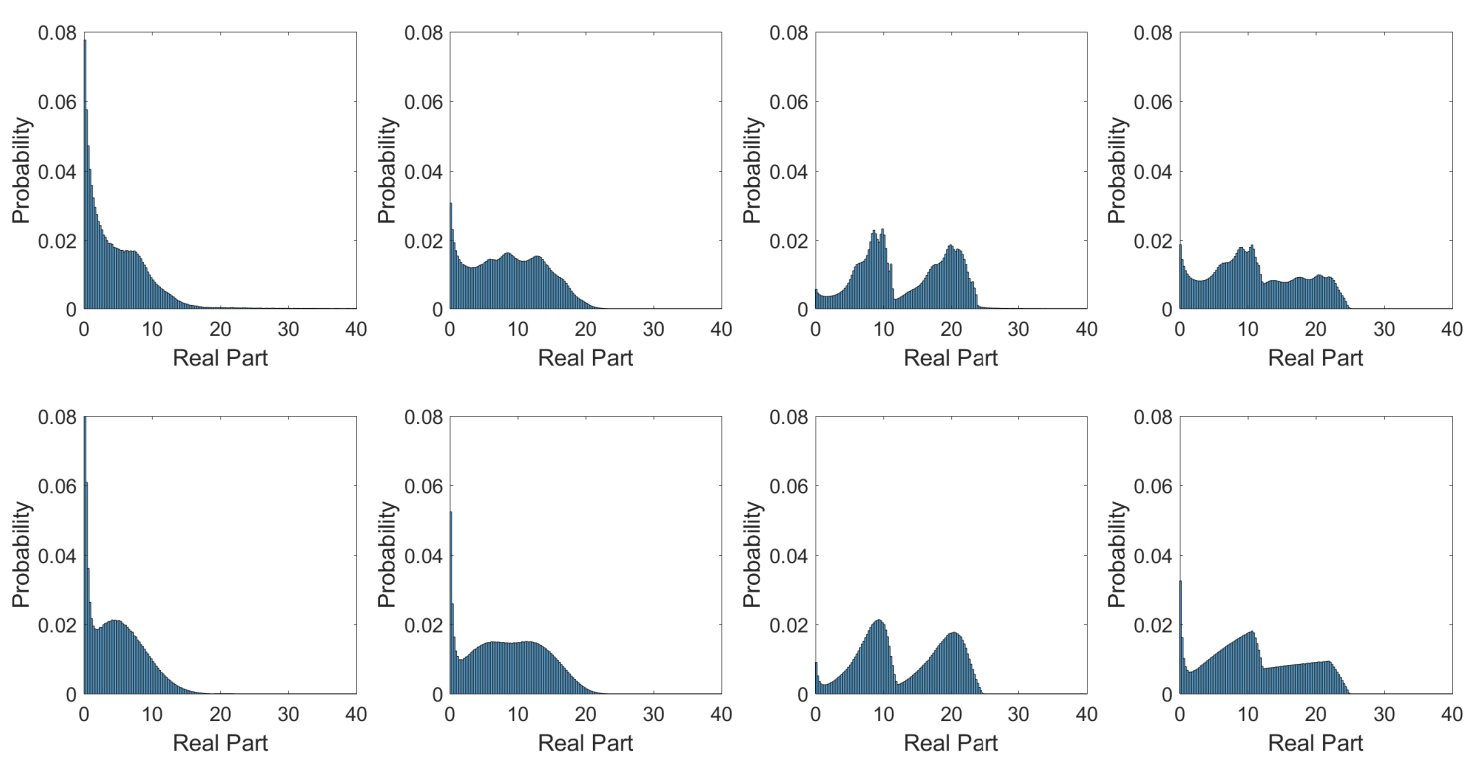

(a)
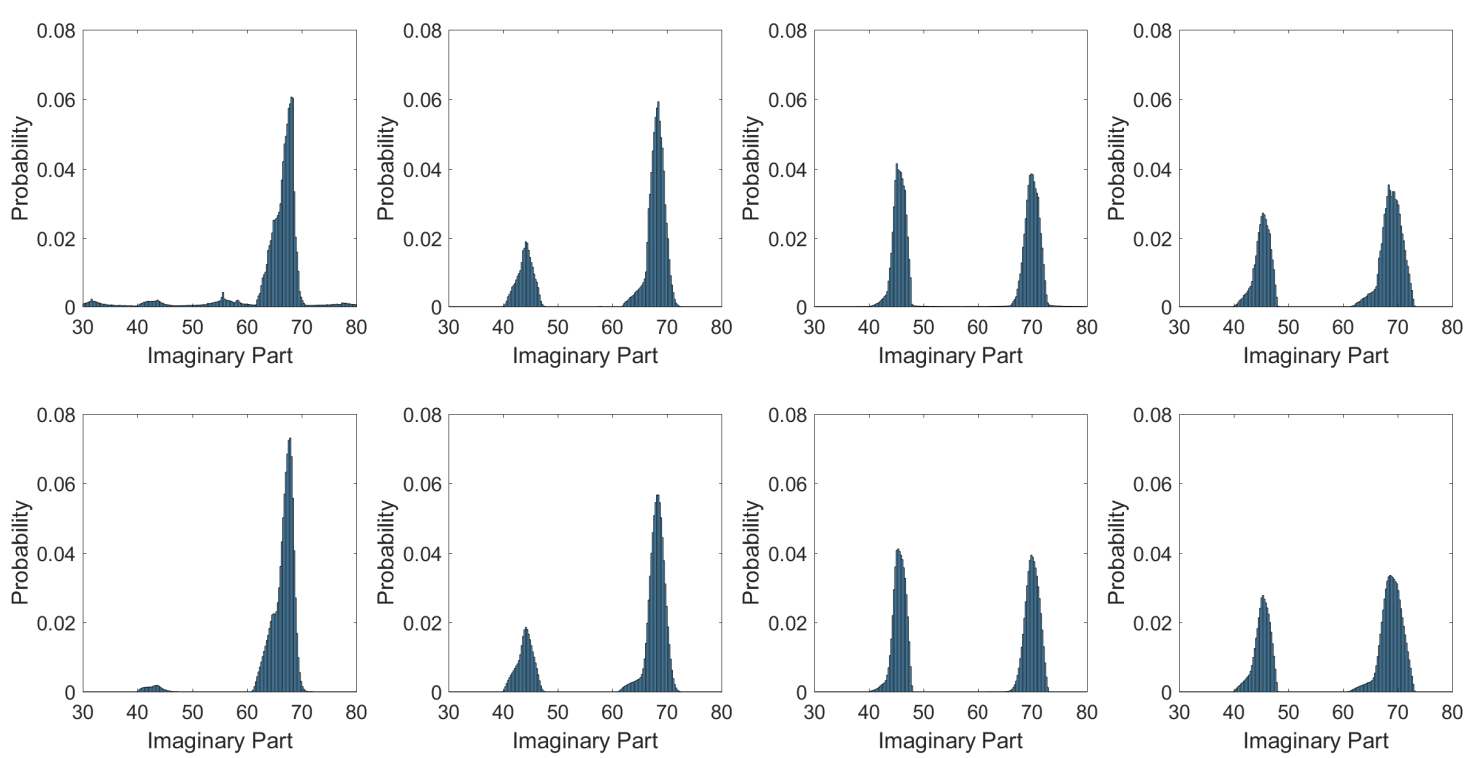

(b)

Figure 5: Occurrences of real (a) and imaginary (b) parts of unstable modes for the first configuration for the surrogate model (first line) and the reference results (second line) and for different distribution laws (from left to right): $\operatorname{Beta}(2,8), \operatorname{Beta}(5,5), \operatorname{Beta}(8,2)$ and Uniform

probability of occurrence that reaches 0.06 (see Figures 6 for configuration 1 ). On the other hand, with a $B(8,2)$ distribution, two unstable modes (at 48 and 70 rad.s ${ }^{-1}$ ) with more or less the same occurrence probability are observed for a $B(8,2)$ distribution. Similar remarks can be inferred from comparison of the repartition of real parts of unstable modes that gives information about the instability growth rate of the unstable modes. For example, the $B(8,2)$ distribution generates low instability growth rate (i.e. the associated real parts take values generally below 10 for configurations 1 and 2 ) whereas the events with greater instability growth rate are significant for a $B(2,8)$ distribution or a uniform law (see Figures 5 and 6 where the values of the real parts are situated between 10 and 20 ).

Finally we can also see a more complex behavior of the second configuration (previously discussed and described in [14]). It is also well reproduced with three unstable pulsations around 50, 60 and 73 rad.s ${ }^{-1}$ 

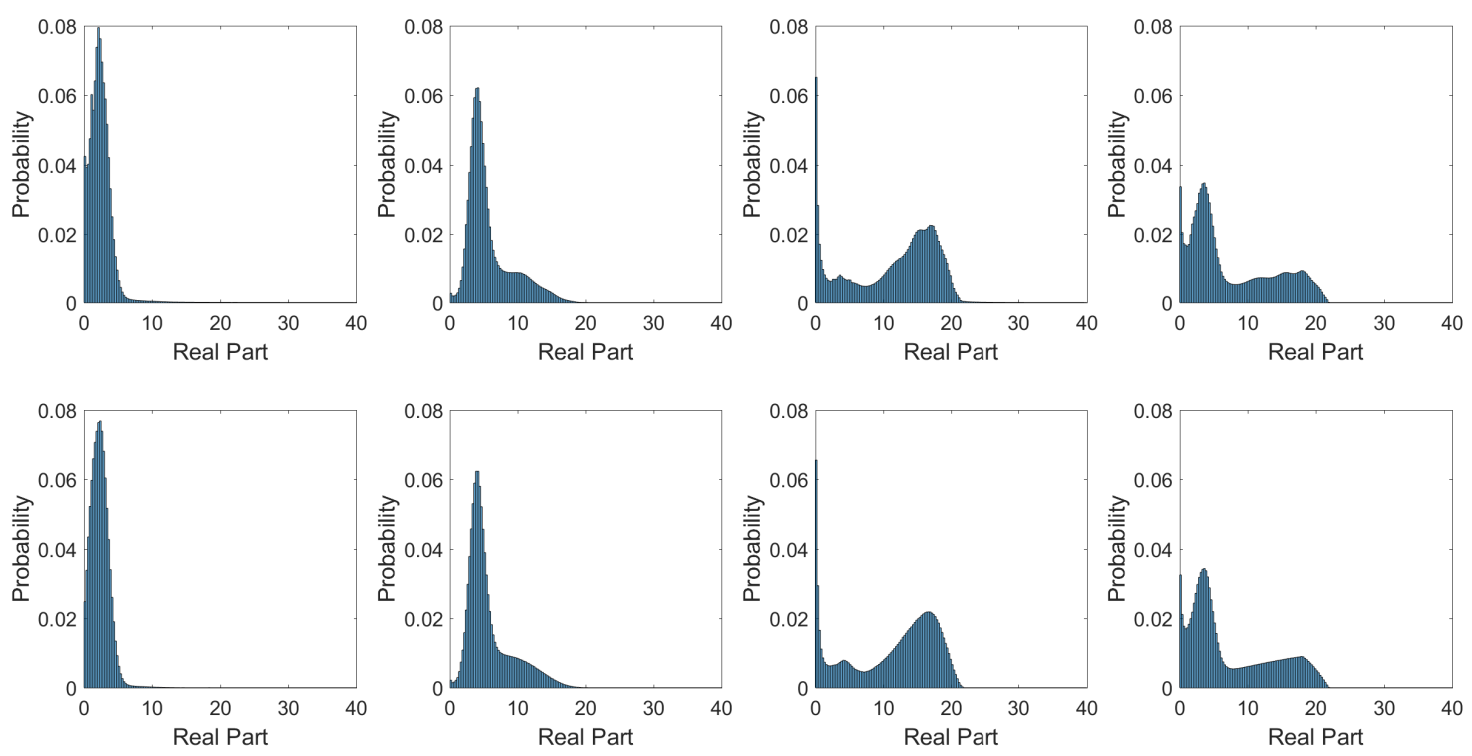

(a)
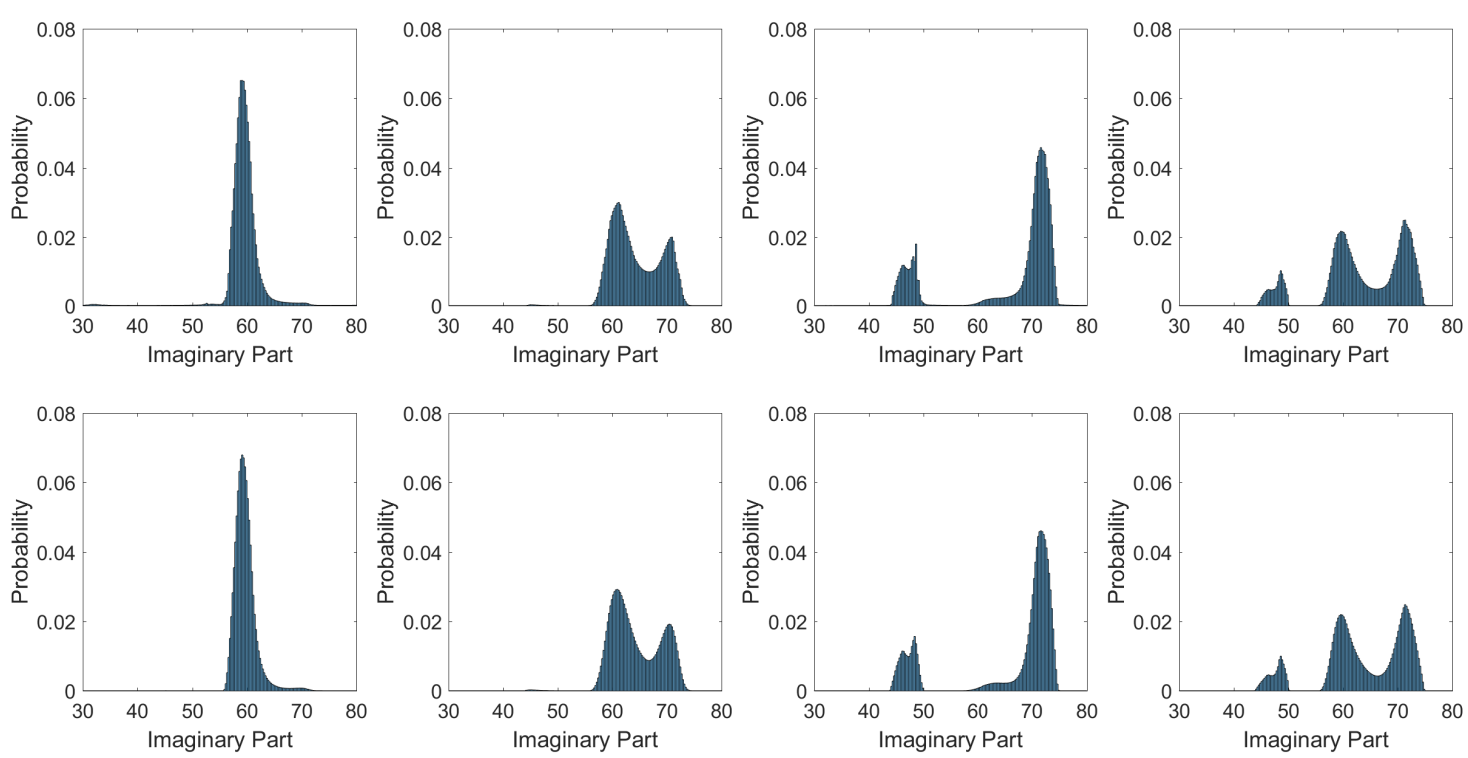

(b)

Figure 6: Occurrences of real (a) and imaginary (b) parts of unstable modes for the second configuration for the surrogate model (first line) and the reference results (second line) and for different distribution laws (from left to right): $\operatorname{Beta}(2,8), \operatorname{Beta}(5,5), \operatorname{Beta}(8,2)$ and Uniform

(with a uniform distribution) whereas only two unstable pulsations around 45 and $70 \mathrm{rad} . \mathrm{s}^{-1}$ are observed for configuration 1.

In conclusion these sets of results tend to demonstrate not only the suitability of the proposed hybrid meta-model to describe the stability behavior of the system but also the non negligible effect of the choice of probability laws on the number of unstable modes, the percentage of stable and unstable occurrences, the frequencies of unstable modes and the instability growth rate. 


\begin{tabular}{cccccccccc}
\hline Eigenvalue & & $\boldsymbol{R e}\left(\boldsymbol{\lambda}_{\mathbf{1}}\right)$ & $\boldsymbol{R e}\left(\boldsymbol{\lambda}_{\mathbf{2}}\right)$ & $\boldsymbol{R e}\left(\boldsymbol{\lambda}_{\mathbf{3}}\right)$ & $\boldsymbol{R e}\left(\boldsymbol{\lambda}_{\mathbf{4}}\right)$ & $\boldsymbol{I m}\left(\boldsymbol{\lambda}_{\mathbf{1}}\right)$ & $\boldsymbol{I m}\left(\boldsymbol{\lambda}_{\mathbf{2}}\right)$ & $\boldsymbol{I m}\left(\boldsymbol{\lambda}_{\mathbf{3}}\right)$ & $\boldsymbol{I m}\left(\boldsymbol{\lambda}_{\mathbf{4}}\right)$ \\
\hline \multirow{2}{*}{ Law B(2,8) $)$} & Coeff 1 & $2.50 \mathrm{E}-5$ & $4.15 \mathrm{E}-4$ & $6.39 \mathrm{E}-4$ & $3.06 \mathrm{E}-5$ & $2.59 \mathrm{E}-5$ & $2.79 \mathrm{E}-4$ & $1.91 \mathrm{E}-4$ & $6.29 \mathrm{E}-6$ \\
& Coeff 2 & $1.38 \mathrm{E}-5$ & $1.32 \mathrm{E}-4$ & $2.18 \mathrm{E}-4$ & $1.21 \mathrm{E}-5$ & $1.21 \mathrm{E}-5$ & $1.16 \mathrm{E}-4$ & $1.25 \mathrm{E}-4$ & $1.28 \mathrm{E}-5$ \\
\multirow{2}{*}{ Law B(5,5)$)$} & Coeff 1 & $2.38 \mathrm{E}-5$ & $4.75 \mathrm{E}-4$ & $5.07 \mathrm{E}-4$ & $1.45 \mathrm{E}-4$ & $2.60 \mathrm{E}-5$ & $2.75 \mathrm{E}-4$ & $1.12 \mathrm{E}-3$ & $1.84 \mathrm{E}-4$ \\
& Coeff 2 & $1.28 \mathrm{E}-5$ & $3.26 \mathrm{E}-4$ & $4.05 \mathrm{E}-4$ & $9.70 \mathrm{E}-5$ & $1.42 \mathrm{E}-5$ & $2.32 \mathrm{E}-4$ & $5.87 \mathrm{E}-4$ & $1.07 \mathrm{E}-4$ \\
\multirow{2}{*}{ Law B(8,2)$)$} & Coeff 1 & $4.61 \mathrm{E}-6$ & $1.35 \mathrm{E}-3$ & $1.37 \mathrm{E}-3$ & $1.56 \mathrm{E}-4$ & $4.90 \mathrm{E}-6$ & $1.39 \mathrm{E}-3$ & $1.20 \mathrm{E}-3$ & $1.55 \mathrm{E}-4$ \\
& Coeff 2 & $7.12 \mathrm{E}-6$ & $7.97 \mathrm{E}-4$ & $7.05 \mathrm{E}-4$ & $8.14 \mathrm{E}-5$ & $4.03 \mathrm{E}-6$ & $6.42 \mathrm{E}-4$ & $6.24 \mathrm{E}-4$ & $8.40 \mathrm{E}-5$ \\
\multirow{2}{*}{ Uniform } & Coeff 1 & $2.12 \mathrm{E}-5$ & $4.73 \mathrm{E}-4$ & $8.99 \mathrm{E}-4$ & $1.45 \mathrm{E}-4$ & $1.97 \mathrm{E}-5$ & $5.86 \mathrm{E}-4$ & $9.72 \mathrm{E}-4$ & $1.19 \mathrm{E}-4$ \\
& Coeff 2 & $1.84 \mathrm{E}-5$ & $8.14 \mathrm{E}-4$ & $8.57 \mathrm{E}-4$ & $1.00 \mathrm{E}-4$ & $1.68 \mathrm{E}-5$ & $8.35 \mathrm{E}-4$ & $1.08 \mathrm{E}-4$ & $8.92 \mathrm{E}-5$ \\
\hline
\end{tabular}

Table 3: Mean square error on first and second polynomial chaos coefficient for each real and imaginary parts of eigenvalue for each considered distribution for the first configuration

\begin{tabular}{cccccccccc}
\hline Eigenvalue & & $\boldsymbol{R e}\left(\boldsymbol{\lambda}_{\mathbf{1}}\right)$ & $\boldsymbol{R e}\left(\boldsymbol{\lambda}_{\mathbf{2}}\right)$ & $\boldsymbol{R e}\left(\boldsymbol{\lambda}_{\mathbf{3}}\right)$ & $\boldsymbol{R e}\left(\boldsymbol{\lambda}_{\mathbf{4}}\right)$ & $\boldsymbol{I m}\left(\boldsymbol{\lambda}_{\mathbf{1}}\right)$ & $\boldsymbol{I m}\left(\boldsymbol{\lambda}_{\mathbf{2}}\right)$ & $\boldsymbol{I m}\left(\boldsymbol{\lambda}_{\mathbf{3}}\right)$ & $\boldsymbol{I m}\left(\boldsymbol{\lambda}_{\mathbf{4}}\right)$ \\
\hline \multirow{2}{*}{ Law B(2,8)$)$} & Coeff 1 & $2.30 \mathrm{E}-4$ & $2.98 \mathrm{E}-3$ & $4.39 \mathrm{E}-3$ & $3.59 \mathrm{E}-3$ & $1.52 \mathrm{E}-4$ & $1.05 \mathrm{E}-3$ & $3.19 \mathrm{E}-3$ & $3.15 \mathrm{E}-3$ \\
& Coeff 2 & $1,81 \mathrm{E}-04$ & $1.17 \mathrm{E}-3$ & $2.88 \mathrm{E}-3$ & $2.86 \mathrm{E}-3$ & $1.20 \mathrm{E}-4$ & $1.79 \mathrm{E}-3$ & $3.36 \mathrm{E}-3$ & $2.03 \mathrm{E}-3$ \\
\multirow{2}{*}{ Law B(5,5)$)$} & Coeff 1 & $6.74 \mathrm{E}-6$ & $2.59 \mathrm{E}-3$ & $3.25 \mathrm{E}-3$ & $3.26 \mathrm{E}-3$ & $2.29 \mathrm{E}-5$ & $5.74 \mathrm{E}-3$ & $5.11 \mathrm{E}-3$ & $3.72 \mathrm{E}-3$ \\
& Coeff 2 & $7.64 \mathrm{E}-6$ & $2.55 \mathrm{E}-3$ & $3.75 \mathrm{E}-3$ & $2.23 \mathrm{E}-3$ & $1.53 \mathrm{E}-5$ & $3.02 \mathrm{E}-3$ & $4.29 \mathrm{E}-3$ & $1.93 \mathrm{E}-3$ \\
\multirow{2}{*}{ Law B(8,2)} & Coeff 1 & $4.55 \mathrm{E}-7$ & $1.41 \mathrm{E}-2$ & $1.41 \mathrm{E}-2$ & $2.07 \mathrm{E}-4$ & $3.33 \mathrm{E}-6$ & $1.59 \mathrm{E}-2$ & $1.59 \mathrm{E}-2$ & $2.10 \mathrm{E}-4$ \\
& Coeff 2 & $3.78 \mathrm{E}-7$ & $5.75 \mathrm{E}-3$ & $5.74 \mathrm{E}-3$ & $3.49 \mathrm{E}-4$ & $2.67 \mathrm{E}-6$ & $6.36 \mathrm{E}-3$ & $6.38 \mathrm{E}-3$ & $3.24 \mathrm{E}-4$ \\
\multirow{2}{*}{ Uniform } & Coeff 1 & $4.45 \mathrm{E}-5$ & $5.86 \mathrm{E}-3$ & $6.01 \mathrm{E}-3$ & $2.99 \mathrm{E}-3$ & $3.02 \mathrm{E}-5$ & $5.37 \mathrm{E}-3$ & $5.52 \mathrm{E}-3$ & $2.38 \mathrm{E}-3$ \\
& Coeff 2 & $7.94 \mathrm{E}-5$ & $8.45 \mathrm{E}-3$ & $8.98 \mathrm{E}-3$ & $1.95 \mathrm{E}-3$ & $4.44 \mathrm{E}-5$ & $8.13 \mathrm{E}-3$ & $8.45 \mathrm{E}-3$ & $1.73 \mathrm{E}-3$ \\
\hline
\end{tabular}

Table 4: Mean square error on first and second polynomial chaos coefficient for each real and imaginary parts of eigenvalue for each considered distribution for the second configuration

\begin{tabular}{|c|c|c|c|c|c|c|}
\hline & \multicolumn{2}{|c|}{$\%$ stable } & \multicolumn{2}{|c|}{$\%$ of one unstable mode } & \multicolumn{2}{|c|}{$\%$ of two unstable modes } \\
\hline & Reference & Meta-Model & Reference & Meta-Model & Reference & Meta-Model \\
\hline Law $B(2,8)$ & 79.678 & 77.719 & 19.774 & 20.290 & 0.548 & 1.987 \\
\hline Law $B(5,5)$ & 10.490 & 10.622 & 60.426 & 60.705 & 29.085 & 28.673 \\
\hline Law $B(8,2)$ & 0.048 & 0.062 & 6.828 & 7.077 & 93.124 & 92.861 \\
\hline Uniform & 30.016 & 30.000 & 29.064 & 29.252 & 40.919 & 40.748 \\
\hline
\end{tabular}

Table 5: Percentage of number of stable or unstable modes for reference and surrogate model for the first configuration

\begin{tabular}{|c|c|c|c|c|c|c|}
\hline & \multicolumn{2}{|c|}{$\%$ stable } & \multicolumn{2}{|c|}{$\%$ of one unstable mode } & \multicolumn{2}{|c|}{$\%$ of two unstable modes } \\
\hline & Reference & Meta-Model & Reference & Meta-Model & Reference & Meta-Model \\
\hline Law $B(2,8)$ & 32.486 & 30.276 & 67.510 & 68.881 & 0.004 & 0.718 \\
\hline Law $B(5,5)$ & 0.840 & 0.784 & 98.915 & 98.995 & 0.245 & 0.221 \\
\hline Law $B(8,2)$ & 0.00 & 0.005 & 74.045 & 73.721 & 25.954 & 26.021 \\
\hline Uniform & 12.673 & 12.569 & 76.235 & 76.588 & 11.092 & 10.843 \\
\hline
\end{tabular}

Table 6: Percentage of number of stable or unstable modes for reference and surrogate model for the second configuration

\subsection{Case of four uncertain parameters: two interval and two random parameters}

The last part of this section is dedicated to the efficiency of the proposed method based on the GPC and the kriging formalisms by considering four uncertain parameters: two interval and two random parameters. As for the previous study the stiffness parameters $k_{11}$ and $k_{22}$ are considered within given intervals $\left[k_{11}^{\min } ; k_{11}^{\max }\right]$ and $\left[k_{22}^{\min } ; k_{22}^{\max }\right]$, respectively and the friction coefficient $\mu$ is taken as random. The uncertain parameter added concerns the stiffness $k_{a}$. Two families of continuous probability distributions are considered: the uniform distribution and three different beta distributions (for more details see Section 4.2). Sets of parameters are 


\begin{tabular}{ccccc}
\hline Type of experimental design & Reference & Roots of polynomial & $\mathbf{3 0 0}$ points LHS & $\mathbf{8 0 0}$ points LHS \\
\hline CEA evaluations & $2.5 \mathrm{E} 7$ & $9 \mathrm{E} 4$ & $8 \mathrm{E} 4$ & $3 \mathrm{E} 4$ \\
\hline
\end{tabular}

Table 7: Number of CEA evaluations for the different methods

given in Table 2.

This study is also carried out to show how to overcome the computational difficulties induced by a high stochastic dimension defined by a large number of random variables. Indeed, considering the construction of the hybrid meta-model, the GPC order is chosen equal to 20 according to the performed convergence study and the multivariate basis is built with the tensor product of uni-dimensional polynomials. A GPC expansion corresponding to the fixed chaos order requires 231 terms to be calculated. The calculating process based on the regression scheme shows a good efficiency in the case of a (uniform-uniform) distribution (i.e. the friction coefficient $\mu$ and the stiffness $k_{a}$ are described via a uniform law) and a (uniform-beta) distribution (i.e. one of the uncertain variables is described by a uniform law, while the other is described by a beta distribution). However, in the case of a (beta-beta) distribution, the associated GPC expansion fails to keep the same performance as the two previous cases due to the use of Jacobi polynomials. Indeed, for high chaos orders, the Fisher matrix issued from the evaluating of the multivariate Jacobi polynomials at the generated experimental design is poorly conditioned. This gives rise to GPC expansions with coefficients that are numerically too high leading to a numerical divergence of the expansions. To cope with this problem, a selection of GPC coefficients is performed. An usual strategy is to perform a truncation by considering that most of models are governed by main effects and low order interactions. In other words, it consists in considering polynomials of low order of interaction [31, 32]. This is carried out by using an hyperbolic norm which helps for the construction of a polynomial basis by keeping high degree polynomials for one variable and low degree polynomials for interactions between variables. Hence, according to this norm which is characterized by a parameter $0<q \leq 1$, the selected polynomials are those for which degrees respects:

$$
\|J\|_{q} \equiv\left(\sum_{i=1}^{r} J_{i}^{q}\right)^{1 / q} \leq m
$$

In this study, $q$ is is chosen equal to 0.6 which leads to the convergence of the expansion while allowing for a strong reduction in the size of the basis(since the associated polynomial basis is composed of only 119 terms). It may be noted that, in the present study, this truncation is used for both Jacobi and Legendre polynomials.

For the GPC construction, two types of experimental designs are considered in the following:

- The first one is composed of roots of polynomials used in the GPC basis. The roots of a polynomial of enough order are used for the uni-dimensional case whereas the multidimensional experimental design is obtained through the tensor product of uni-dimensional experimental design. Here, uni-dimensional experimental designs are the roots of the $30^{t h}$ polynomial of the basis. So the final experimental designs associated to the GPC development is so composed of 900 samples;

- The second kind of experimental design used corresponds to the well-known stratified sampling called LHS. It is well-known that the choice of sample size is a practical difficulty when applying LHS. The sample size to give adequate statistics can not be determined a priori: large sample size may be both extremely time-consuming and costly in terms of storage requirements, while small sample size may not give acceptable statistical results. So in the following, we propose to investigate the impact of the number of LHS sample points by generating two LHS with 800 and 300 samples, respectively.

Considering the experimental design associated to kriging, The LHS with 100 sample points will be used. As a reminder, reference results are obtained with $2.510^{7}$ CEA evaluations. The number of CEA calculations required for the different experimental designs are summed up in Table 7.

First of all, a scanning/MCS is performed in order to obtain the reference results (i.e. the percentage of stable and unstable occurrences, the frequencies of unstable modes and the instability growth rate). In this perspective, a regular grid of $50 * 50=2500$ sample points for stiffnesses $k_{11}$ and $k_{22}$ is built. For each possible distribution of $\left(\mu-k_{a}\right), 10000$ LHS sample points are used. So distributions of real and imaginary parts of eigenvalues are obtained by performing CEA analysis via the $2500 * 10000=2.510^{7}$ sample points.

For each random variable (i.e. the friction coefficient $\mu$ and the stiffness $k_{a}$ ), four distribution laws are tested (a uniform distribution and the $B(5,5), B(2,8)$ and $B(8,2)$ distributions) inducing a total of 16 types of distribution laws for the pair of independent random variables $\mu$ and $k_{a}$. Occurrences of real and imaginary 

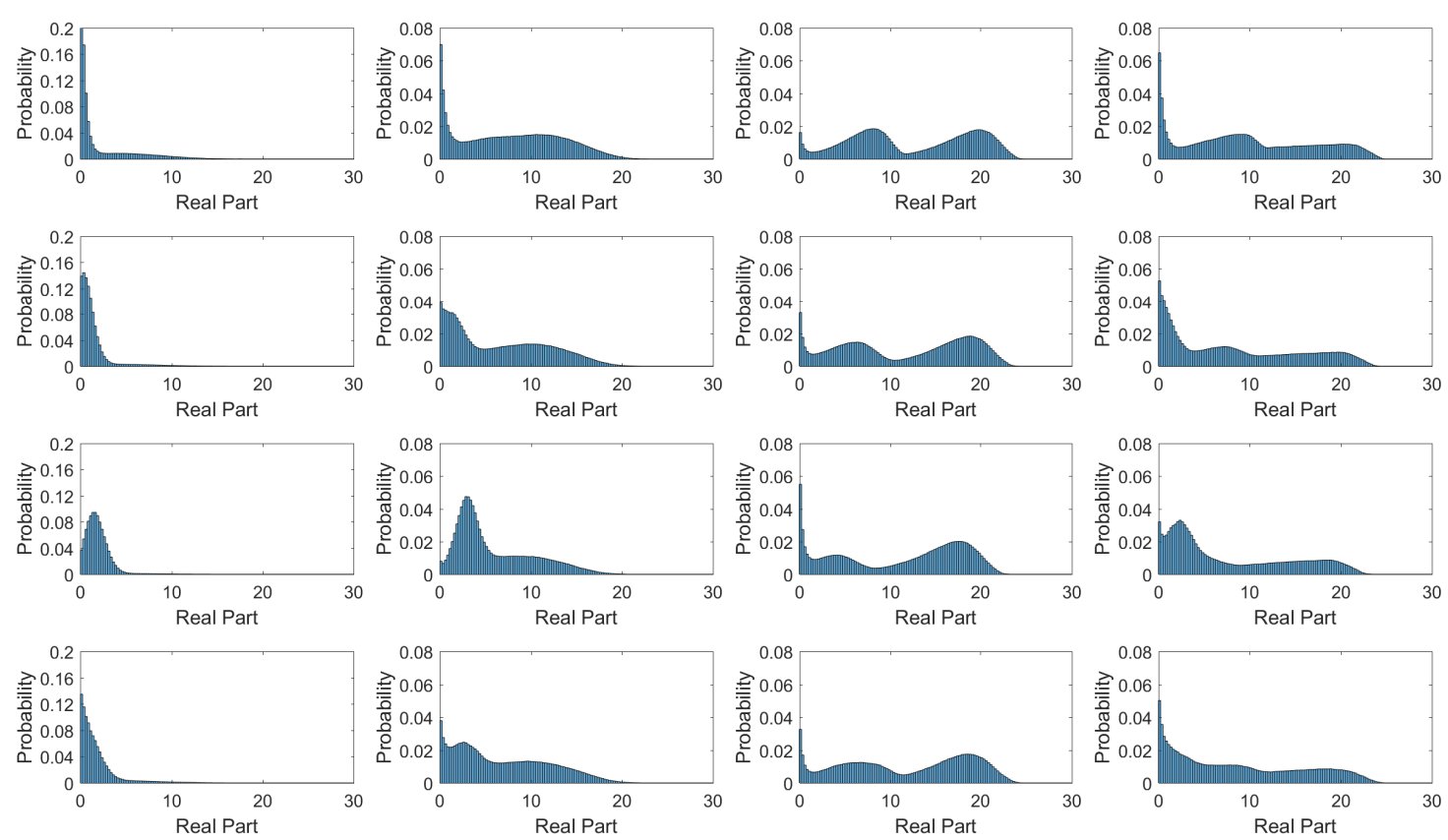

Figure 7: Occurrences of real parts of unstable modes for the reference case for different distributions for $\mu$ and $k_{a}$ : from the left column to the right column: $\mathrm{B}(2,8), \mathrm{B}(5,5), \mathrm{B}(8,2)$ and Uniform distribution for $\mu$ and from first line to last line: $\mathrm{B}(2,8), \mathrm{B}(5,5), \mathrm{B}(8,2)$ and Uniform distribution for $k_{a}$

parts of unstable modes are displayed in Figures 7 and 8 , respectively. To facilitate the reading of the results, the figures are composed of $4 \times 4$ sub-figures : each column corresponds to a distribution law for $\mu$ and each line to a distribution law for $k_{a}$.

Secondly, Figures 9 and 10 (Figures 11 and 12, respectively) illustrate the results by using the hybrid metamodel with the selection based on the roots of polynomial (the LHS with 300 samples, respectively). Results based on the LHS with 800 samples are not displayed since they are very similar to the results presented in Figures 9 and 10. Then Table 8 gives the number of unstable modes and the percentage of stable and unstable occurrences. All the numerical results based on the meta-model are in perfect agreement with the reference results. The number of unstable modes and the percentage of stable and unstable occurrences are very well predicted in all configurations (see Table 8). Then, unstable frequencies around 48, 58 and $70 \mathrm{rad} . \mathrm{s}^{-1}$ are well detected. It can also be noted that the unstable frequency at $48 \mathrm{rad} . \mathrm{s}^{-1}$ that barely exists in the case of a $\mathrm{B}(5,5)$ distribution for $\mu$ and $\mathrm{B}(8,2)$ for $k_{a}$ is also well reproduced. By comparing the reference results and the meta-model results, occurrences of the associated positive real parts are also very similar. Moreover the influence of distribution laws for $\mu$ and $k_{a}$ is well reproduced. A modification of the distribution of $\mu$ has a stronger influence than a modification of the distribution of $k_{a}$ on the evolutions of the pulsation of unstable modes. Indeed, whatever the law of probability associated with $k_{a}$, the pulsation of unstable modes are within the interval $[55,72]$ rad.s $\mathrm{s}^{-1}$ (with a peak at $60 \mathrm{rad} . \mathrm{s}^{-1}$ ) in the case of a $\mathrm{B}(2,8)$ distribution for $\mu$ while three groups of unstable modes are present (localized around 48,60 and $72 \mathrm{rad} . \mathrm{s}^{-1}$ ) with a uniform distribution for $\mu$. However, the effects of the different distribution laws for $k_{a}$ exists for a given distribution law of $\mu$ as indicated in Figures 9, 10, 11 and 12. So the stability behavior of the system is accurately represented via the proposed methodology based on the coupling of kriging and GPC expansion.

Finally results based on a small LHS sample size with only 300 points are provided. Focusing on the prediction of the number of unstable modes and the associated percentage of stable and unstable occurrences, the numerical results agree very well with the reference results. Now, considering the occurrences of real and imaginary parts of unstable modes, it is noted that the global stability behavior of the system is well estimated even if sometimes small differences are observed. All the frequency intervals of unstable pulsations are well detected and the associated real parts are faithfully predicted for a non-negligible lower computation cost. It may be noted that an even smaller number of samples will necessarily lead to a deterioration of the results. This illustrates one of the difficulties in using LHS which is the a priori choice of the sample size to give adequate statistics. 

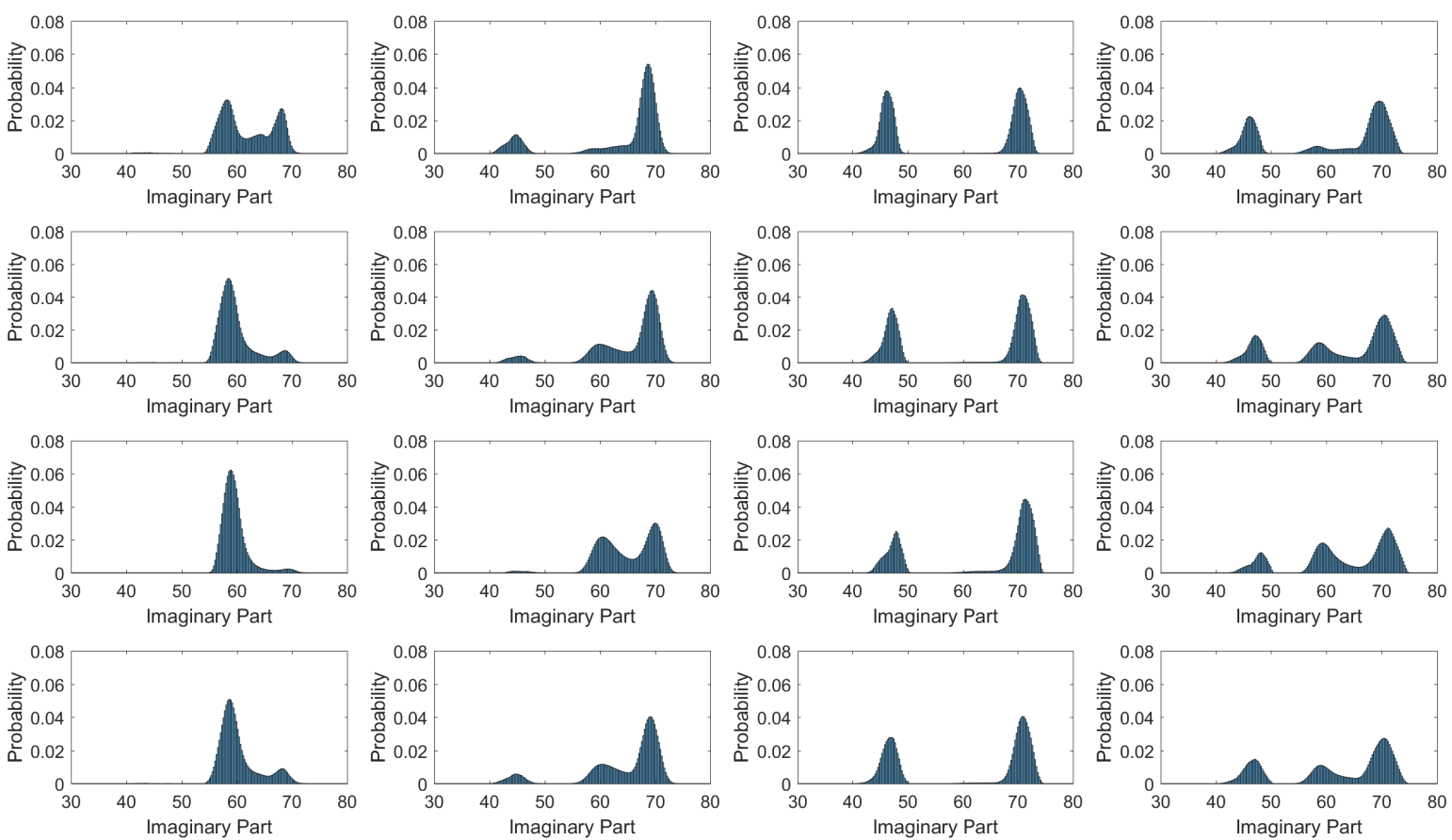

Figure 8: Occurrences of imaginary parts of unstable modes for the reference case for different distributions for $\mu$ and $k_{a}$ : from the left column to the right column: $\mathrm{B}(2,8), \mathrm{B}(5,5), \mathrm{B}(8,2)$ and Uniform distribution for $\mu$ and from first line to last line: $\mathrm{B}(2,8), \mathrm{B}(5,5), \mathrm{B}(8,2)$ and Uniform distribution for $k_{a}$

Concerning the impact of the chosen probability laws on the results, the remarks previously stated in Section 4.3 remain valid: the number of unstable modes, the percentage of stable and unstable occurrences, the evolution of unstable frequencies and the instability growth rate are drastically very dependent on the chosen probability laws.

\begin{tabular}{|c|c|c|c|c|c|c|c|c|c|c|c|c|c|}
\hline \multicolumn{2}{|c|}{ Distributions } & \multicolumn{4}{|c|}{$\%$ Stable } & \multicolumn{4}{|c|}{$\%$ of one unstable mode } & \multicolumn{4}{|c|}{$\%$ of two unstable modes } \\
\hline$\mu$ & $\mathbf{k}_{\mathrm{a}}$ & Ref & $\begin{array}{l}\text { Roots } \\
\text { Grid }\end{array}$ & $\begin{array}{l}\text { LHS } \\
300\end{array}$ & $\begin{array}{c}\text { LHS } \\
800\end{array}$ & Ref & $\begin{array}{c}\text { Roots } \\
\text { Grid }\end{array}$ & $\begin{array}{c}\text { LHS } \\
300\end{array}$ & $\begin{array}{c}\text { LHS } \\
800\end{array}$ & Ref & $\begin{array}{c}\text { Roots } \\
\text { Grid }\end{array}$ & $\begin{array}{c}\text { LHS } \\
300\end{array}$ & $\begin{array}{l}\text { LHS } \\
800\end{array}$ \\
\hline $\mathrm{B}(2,8)$ & $\mathrm{B}(2,8)$ & 61.76 & 60.70 & 48.45 & 61.42 & 38.06 & 38.55 & 49.61 & 38.31 & 0.18 & 0.71 & 1.94 & 0.27 \\
\hline $\mathrm{B}(2,8)$ & $\mathrm{B}(5,5)$ & 33.36 & 33.02 & 33.50 & 34.22 & 66.58 & 66.40 & 66.43 & 65.73 & 0.06 & 0.50 & 0.06 & 0.05 \\
\hline $\mathrm{B}(2,8)$ & $\mathrm{B}(8,2)$ & 30.41 & 29.03 & 26.15 & 29.16 & 69.59 & 70.40 & 72.21 & 70.61 & 0.01 & 0.49 & 1.62 & 0.23 \\
\hline $\mathrm{B}(2,8)$ & Uniform & 42.25 & 41.09 & 37.36 & 41.96 & 57.66 & 58.28 & 60.51 & 57.77 & 0.09 & 0.55 & 2.00 & 0.27 \\
\hline $\mathrm{B}(5,5)$ & $\mathrm{B}(2,8)$ & 6.83 & 7.09 & 7.28 & 7.34 & 75.05 & 73.07 & 73.58 & 73.09 & 18.12 & 19.84 & 19.14 & 19.58 \\
\hline $\mathrm{B}(5,5)$ & $\mathrm{B}(5,5)$ & 1.72 & 2.31 & 2.45 & 2.24 & 91.40 & 89.70 & 89.96 & 89.92 & 6.88 & 7.99 & 7.60 & 7.83 \\
\hline $\mathrm{B}(5,5)$ & $\mathrm{B}(8,2)$ & 0.77 & 0.80 & 0.75 & 0.75 & 97.68 & 97.53 & 97.30 & 97.44 & 1.55 & 1.68 & 1.94 & 1.81 \\
\hline $\mathrm{B}(5,5)$ & Uniform & 3.15 & 3.47 & 3.33 & 3.57 & 87.67 & 86.44 & 86.46 & 85.99 & 9.18 & 10.09 & 10.20 & 10.44 \\
\hline $\mathrm{B}(8,2)$ & $\mathrm{B}(2,8)$ & 3.15 & 3.47 & 3.33 & 3.57 & 87.67 & 86.44 & 86.46 & 85.99 & 9.18 & 10.09 & 10.20 & 10.44 \\
\hline $\mathrm{B}(8,2)$ & $\mathrm{B}(5,5)$ & 0.01 & 0.02 & 0.01 & 0.02 & 29.81 & 28.05 & 28.43 & 28.43 & 70.19 & 71.93 & 71.56 & 71.55 \\
\hline $\mathrm{B}(8,2)$ & $\mathrm{B}(8,2)$ & 0.00 & 0.00 & 0.01 & 0.01 & 53.92 & 54.74 & 48.40 & 54.08 & 46.07 & 45.25 & 51.60 & 45.91 \\
\hline $\mathrm{B}(8,2)$ & Uniform & 0.02 & 0.02 & 0.02 & 0.02 & 32.87 & 31.94 & 31.81 & 32.33 & 67.11 & 68.03 & 68.16 & 67.65 \\
\hline Uniform & $\mathrm{B}(2,8)$ & 23.42 & 23.83 & 20.72 & 23.39 & 41.76 & 40.17 & 42.14 & 40.82 & 34.83 & 36.00 & 37.07 & 35.79 \\
\hline Uniform & $\mathrm{B}(5,5)$ & 13.11 & 13.72 & 12.83 & 13.66 & 60.98 & 59.32 & 60.59 & 59.53 & 25.91 & 26.97 & 26.58 & 26.80 \\
\hline Uniform & $\mathrm{B}(8,2)$ & 11.93 & 11.97 & 10.19 & 11.79 & 71.04 & 70.77 & 72.57 & 71.60 & 17.02 & 17.26 & 17.22 & 16.62 \\
\hline Uniform & Uniform & 16.22 & 16.40 & 13.24 & 16.11 & 57.66 & 56.92 & 58.32 & 57.30 & 26.12 & 26.68 & 28.32 & 26.59 \\
\hline
\end{tabular}

Table 8: Number of unstable modes and percentage of stable and unstable occurrences for the reference and the different kriging meta-models for the different configurations 

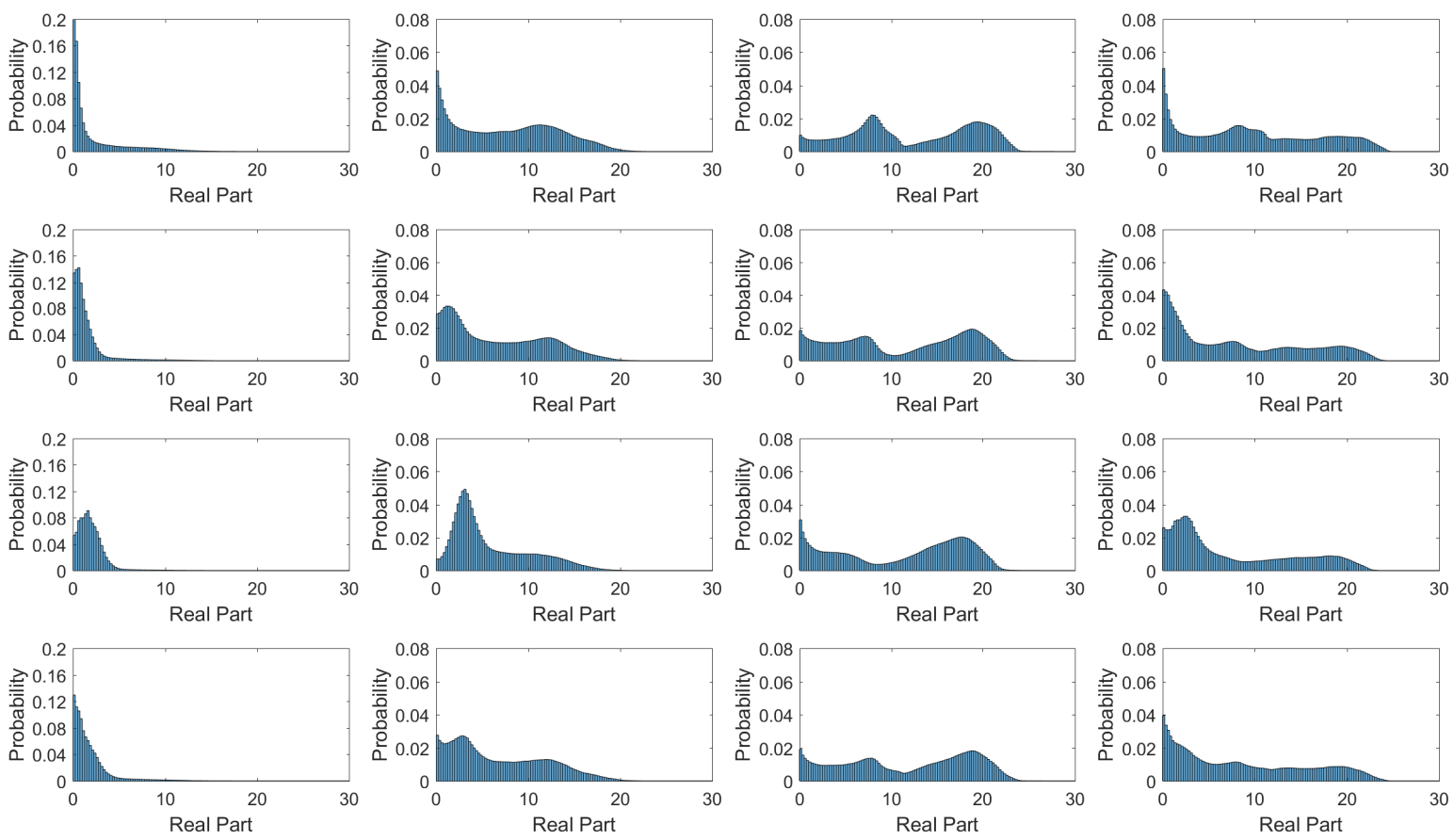

Figure 9: Occurrences of real parts of unstable modes for the roots grid case for different distributions for $\mu$ and $k_{a}$ : from the left column to the right column: $\mathrm{B}(2,8), \mathrm{B}(5,5), \mathrm{B}(8,2)$ and Uniform distribution for $\mu$ and from first line to last line: $\mathrm{B}(2,8), \mathrm{B}(5,5), \mathrm{B}(8,2)$ and Uniform distribution for $k_{a}$
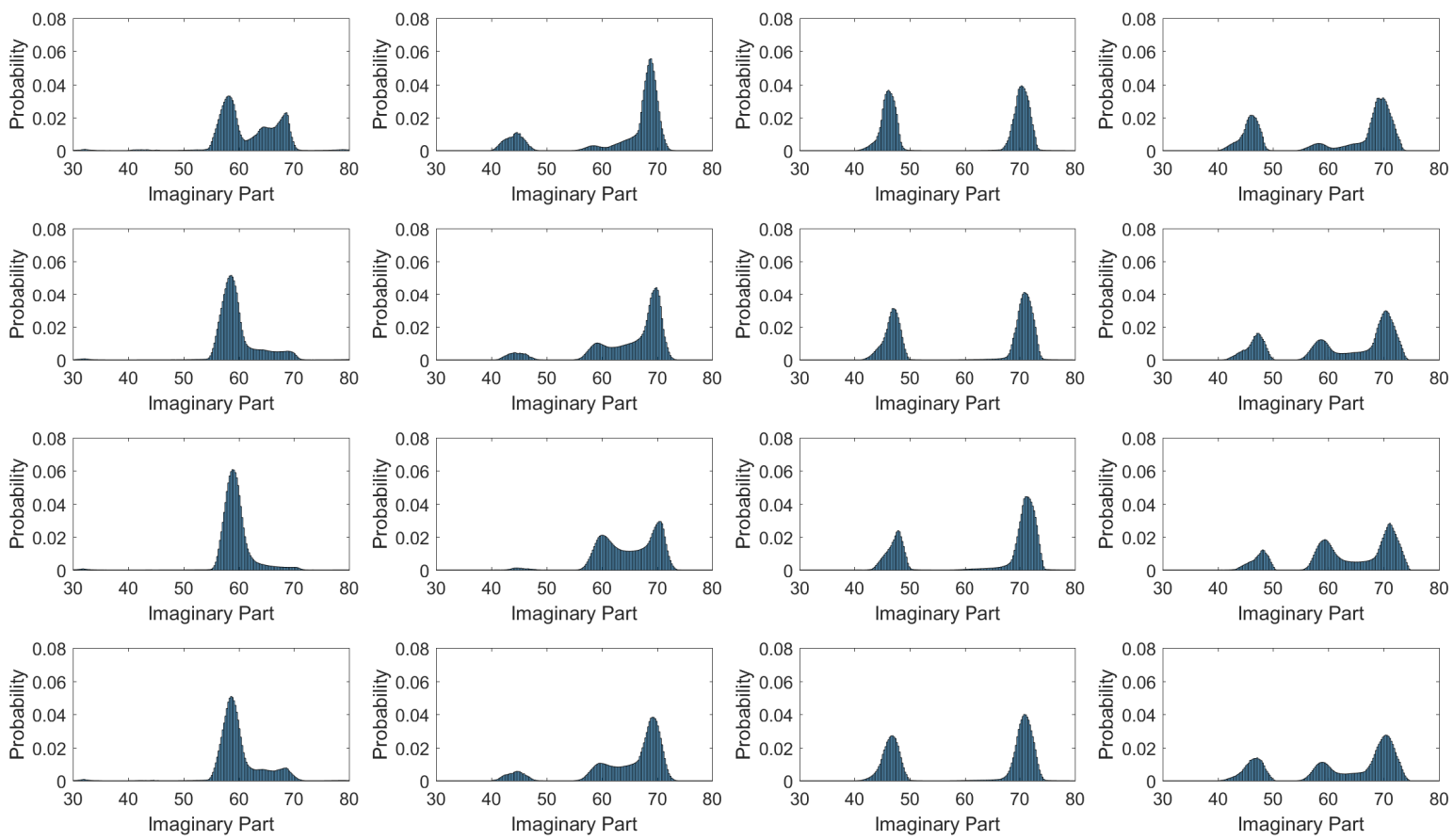

Figure 10: Occurrences of imaginary parts of unstable modes for the roots grid case for different distributions for $\mu$ and $k_{a}$ : from the left column to the right column: $\mathrm{B}(2,8), \mathrm{B}(5,5), \mathrm{B}(8,2)$ and Uniform distribution for $\mu$ and from first line to last line: $\mathrm{B}(2,8), \mathrm{B}(5,5), \mathrm{B}(8,2)$ and Uniform distribution for $k_{a}$ 

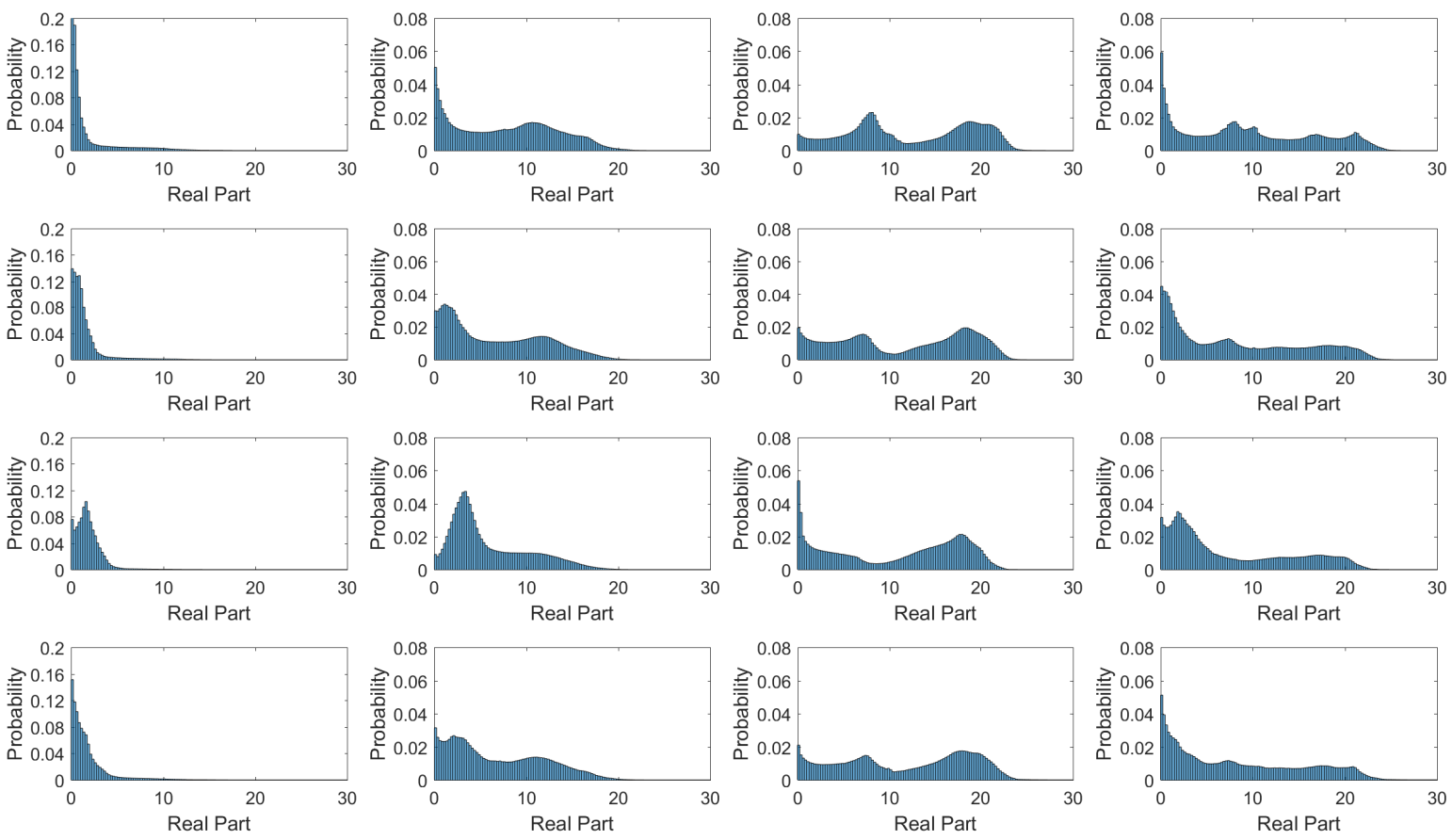

Figure 11: Occurrences of real parts of unstable modes for the LHS 300 case for different distributions for $\mu$ and $k_{a}$ : from the left column to the right column: $\mathrm{B}(2,8), \mathrm{B}(5,5), \mathrm{B}(8,2)$ and Uniform distribution for $\mu$ and from first line to last line: $\mathrm{B}(2,8), \mathrm{B}(5,5), \mathrm{B}(8,2)$ and Uniform distribution for $k_{a}$
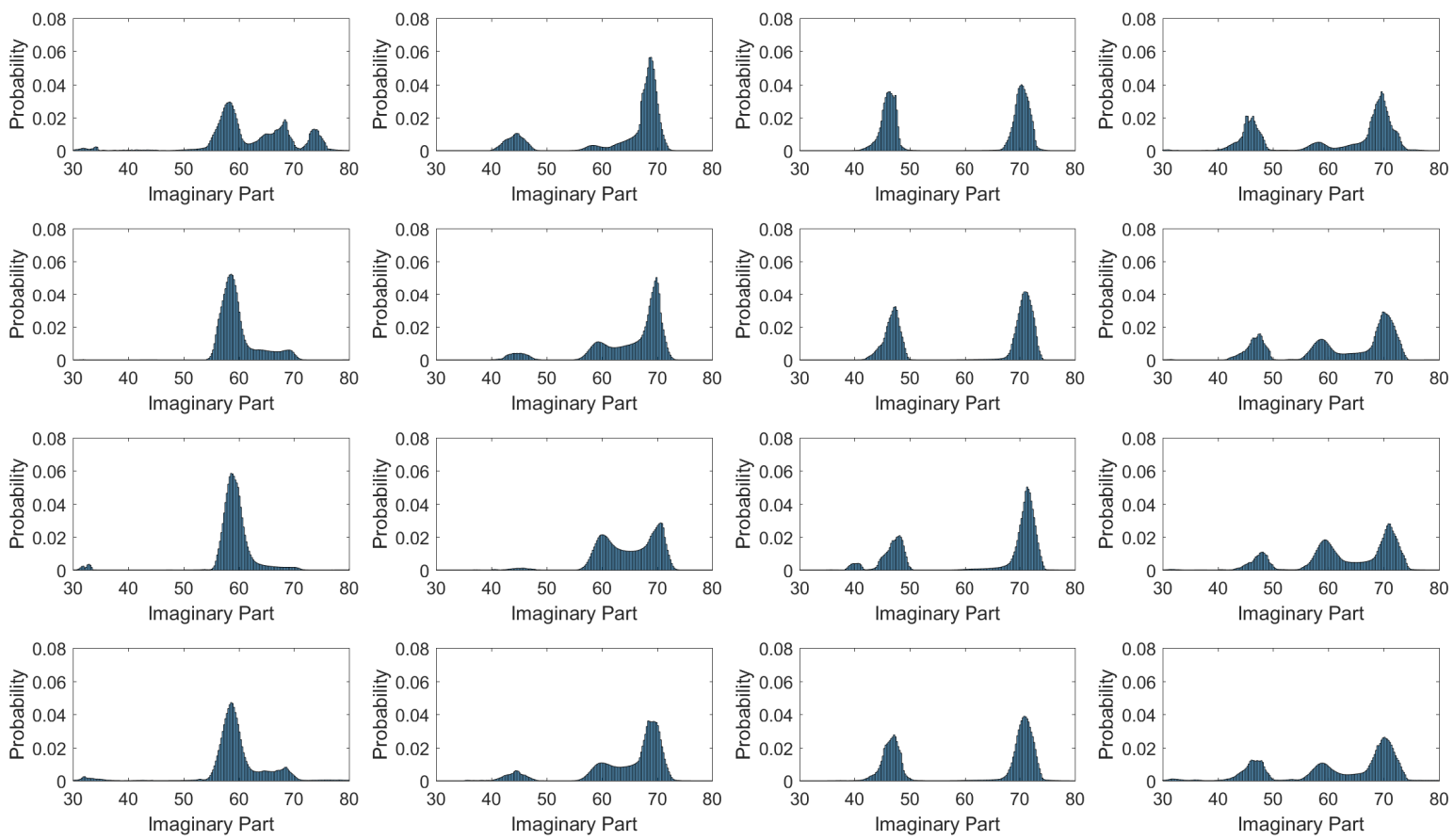

Figure 12: Occurrences of imaginary parts of unstable modes for the LHS 300 case for different distributions for $\mu$ and $k_{a}$ : from the left column to the right column: $\mathrm{B}(2,8), \mathrm{B}(5,5), \mathrm{B}(8,2)$ and Uniform distribution for $\mu$ and from first line to last line: $\mathrm{B}(2,8), \mathrm{B}(5,5), \mathrm{B}(8,2)$ and Uniform distribution for $k_{a}$ 


\section{Conclusion}

The propagation and the quantification of uncertainty is nowadays a crucial point in the design of a mechanical system, including in the field of friction-induced vibrations. In the present study, a non-intrusive stochastic approach based on the GPC and the kriging meta-models is tested to determine whether it is possible to improve the efficiency of meta-modeling (in regards to the issue of computational time consuming and storage) for instability predictions of mechanical system incorporating uncertainties of different natures.

Uncertainty quantification of instability via surrogate modeling is performed on a self-excited four-dof friction system. The proposed methodology seems to be a useful tool in order to give an efficient hybrid meta-model and to provide valuable information regarding not only the number of unstable modes and the percentage of stable and unstable occurrences but also the output distribution for frequencies of unstable modes and the instability growth rate.

Some non-exhaustive list of interesting further developments and studies can be considered:

- In the present study efficiency of the proposed methodology is addressed to the problem of friction-induced vibration which corresponds just as one of many possible examples of application. It is worth noting that this method can be employed on other systems and is not dedicated or dependent to the case of frictioninduced instabilities.

- The applicability of the proposed approach for a full brake system will be tested in a future study in order to predict the occurrence of squeal events. Moreover future developments have now to be undertaken from the practical view point in order to facilitate finding design solutions for the industrial problems in the field of friction-induced vibration and noise. Even if the proposed strategy represents a drastic reduction of computation costs in the present case (3000 CEA to be compared to the $2.510^{7} \mathrm{CEA}$ required for the reference obtained with a scanning/MCS approach), carrying out a non-negligible number of CEA calculations even with a reduced finite element model of a full brake system is still questionable. So it would be interesting in a future work to focus on a strategy to reduce more the number of CEA required which can be strongly related to the high order of the GPC and the related calculations.

- It is also well known that the stability analysis about a static sliding equilibrium point may lead to an under-estimation or an over-estimation of the unstable modes observed in the non-linear computational simulation. More realistic results and information about non-linear phenomena such as periodic, quasiperiodic and chaotic vibrations can be predicted only by considering the original non-linear system and primary role of the non linearity encountered in friction-induced vibrations. Future studies should also focus on a better understanding and modeling of non-linear phenomena in order to predict friction-induced vibrations and noise by comparison with experimental tests.

- The question of the strategy to be adopted in practice, in the case of using a finite element model of brake system, between conducting deterministic simulations in the time domain or performing CEA is also a completely open and sensitive issue. Indeed, conducting time simulations for a limited number of cases allows to estimate the transient and stationary responses that could provide additional and more reliable information for the specific chosen deterministic cases. However, this strategy does not allow to have valuable information on the non-negligible sensitivity of the stability property of the self-excited frictional system versus the variations of design parameters (due to the limited number of time simulations that can be performed within a reasonable time). So it may be preferable to use hybrid meta-modeling approach to propagate and quantify uncertainties in friction-induced instabilities even if this strategy allows only to define a robust design for the propensity of instability to the variations of many parameters. From a more general point of view, these two visions are complementary, each one having advantages and disadvantages.

- Finally one of the most crucial next step can be to be able to propose more practical and commonly implemented techniques for engineering applications.

\section{Acknowledgments}

This work was achieved within PSA Peugeot Citroën Stellab program - OpenLab Vibro-Acoustic- Tribology@Lyon (VAT@Lyon).

J.-J. Sinou acknowledges the support of the Institut Universitaire de France. 


\section{References}

[1] T. Bultin and J. Woodhouse. Sensitivity of friction-induced vibration in idealised systems. Journal of Sound and Vibration, 319(1-2):182-198, 2009.

[2] T. Bultin and J. Woodhouse. Friction-induced vibration: quantifying sensitivity and uncertainty. Journal of Sound and Vibration, 329(1-2):509-526, 2010.

[3] S. Oberst and J. C. S. Lai. Statistical analysis of brake squeal noise. Journal of Sound and Vibration, 330:2978-2994, 2011.

[4] A. Culla and F. Massi. Uncertainty model for contact instability prediction. Journal of the Acoustical Society of America, 126:1111-1119, 2009.

[5] L. Nechak, S. Berger, and E. Aubry. Non-intrusive generalized polynomial chaos for the stability analysis of uncertain dynamic friction systems. Journal of Sound and Vibration, 332(5):1204-1215, 2013.

[6] E. Sarrouy, O. Dessombz, and J.-J. Sinou. Piecewise polynomial chaos expansion with an application to brake squeal of a linear brake system. Journal of Sound and Vibration, 332:577-594, 2013.

[7] Z. Zhang, S. Oberst, and J. C. S. Lai. On the potential of uncertainty analysis for prediction of brake squeal propensity. Journal of Sound and Vibration, 377(1):123-132, 2013.

[8] F. Massa, H.Q. Do, T. Tison, and O. Casier. Uncertain friction-induced vibration study: Coupling of fuzzy logic, fuzzy sets, and interval theories. ASME Journal of Risk and Uncertainty-Part B, 1(1-2):12 pages, 2015.

[9] H. Lu and D. Yu. Brake squeal reduction of vehicle disc brake system with interval parameters by uncertain optimization. Journal of Sound and Vibration, 333(26):7313-7325, 2014.

[10] G. Matheron. Principles of geostatistics. Economic geology, 58(8):1246-1266, 1963.

[11] J. Sacks, W.J. Welch, T.J. Mitchell, and H.P. Wynn. Design and analysis of computer experiments. Statistical science, pages 409-423, 1989.

[12] A. Nobari, H. Ouyang, and P. Bannister. Uncertainty quantification of squeal instability via surrogate modelling. Mechanical Systems and Signal Processing, 60-61:887-908, 2015.

[13] L. Nechak, F. Gillot, S. Besset, and J-J. Sinou. Sensitivity analysis and kriging based models for robust stability analysis of brake systems. Journal of Mechanical Research Communication, 69(4):2017-2023, 2015.

[14] E. Denimal, L. Nechak, S. Nacivet, and J-J. Sinou. Kriging surrogate models for predicting the complex eigenvalues of mechanical systems subjectid to friction-induced vibration. Shock and vibration, 2016.

[15] M. Treimer, B. Allert, K. Dylla, and G. Müller. Uncertainty quantification applied to the mode coupling phenomenon. Journal of Sound and Vibration, 333:171-187, 2014.

[16] H. Lu, W.B. Shangguan, and D. Yu. A unified approach for squeal instability analysis of disc brakes with two types of random-fuzzy uncertainties. Mechanical Systems and Signal Processing, 93:281-298, 2016.

[17] S.N. Lophaven, H.B. Nielsen, and J. Sondergaard. Dace a Matlab Kriging Toolbox. Technical report, Technical University of Denmark, 122002.

[18] http://www.esteco.com.

[19] P. Kersaudy, B. Sudret, N. Varsier, O. Picon, and J. Wiart. A new surrogate modeling technique combining kriging and polynomial chaos expansions-application to uncertainty analysis in computational dosimetry. Journal of Computational Physics, 286:103-117, 2015.

[20] N. Wiener. The homogeneous chaos. American Journal of Mathematics, 60(4):897-936, 1938.

[21] D. Xiu and G. Karniadakis. The wiener-askey polynomial chaos for stochastic differential equations. SIAM journal on scientific computing, 24(2):619-644, 2002.

[22] D. Xiu and G. Karniadakis. Modeling uncertainty in flow simulations via generalized polynomial chaos. Journal of computational physics, 187(1):137-167, 2003.

[23] R. Ghanem and P.D. Spanos. Stochastic finite elements: A spectral approach. Springer Verlag, 1991.

[24] M. Pellissetti and R. Ghanem. Iterative solution of systems of linear equations arising in the context of stochastic finite elements. Advances in Engineergin Software, 31(8):607-616, 2000.

[25] M. Berveiller, B. Sudret, and M. Lemaire. Stochastic finite element: a non intrusive approach by regression. European Journal of Computational Mechanics/Revue Européenne de Mécanique Numérique, 15(1-3):81-92, 2006.

[26] M.D. McKay, R.J. Beckman, and W.J. Conover. A comparison of three methods for selecting values of input variables in the analysis of output from a computer code. Technometrics, 42(1):55-61, 2000.

[27] V.V. Fedorov. Theory of optimal experiments. Elsevier, 1972.

[28] A. Atkinson, A. Donev, and R. Tobias. Optimum experimental designs, with SAS, volume 34 . Oxford University Press, 2007.

[29] B. Sudret. Global sensitivity analysis using polynomial chaos expansions. Reliability Engineering EJ System Safety, 93(7):964979, 2008.

[30] J. Hulten. Friction phenomena related to drum brake squeal instabilities. In Proc. ASME Design Eng. Tech. Conf. 16th Biennial Conf. Mech. Vib. \& Noise, 1997.

[31] G. Blatman and B. Sudret. Adaptive sparse polynomial chaos expansion based on least angle regression. Journal of Computational Physics, 230(6):2345-2367, 2011.

[32] D.C. Montgomery. Design and analysis of experiments. John Wiley \& Sons, 2008. 